\title{
A reassessment of the Burns temperature and its relationship to the diffuse scattering, lattice dynamics, and thermal expansion in the relaxor $\mathrm{Pb}\left(\mathrm{Mg}_{1 / 3} \mathrm{Nb}_{2 / 3}\right) \mathrm{O}_{3}$
}

\author{
P. M. Gehring, ${ }^{1}$ H. Hiraka, ${ }^{2,3}$ C. Stock,${ }^{4}$ S.-H. Lee, ${ }^{1}$ W. Chen, ${ }^{5}$ Z.-G. Ye, ${ }^{5}$ S. B. Vakhrushev,${ }^{6}$ and Z. Chowdhuri ${ }^{1}$ \\ ${ }^{1}$ NIST Center for Neutron Research, National Institute of Standards and Technology, Gaithersburg, Maryland 20899-6100, USA \\ ${ }^{2}$ Department of Physics, Brookhaven National Laboratory, Upton, New York 11973-5000, USA \\ ${ }^{3}$ Institute for Materials Research, Tohoku University, Sendai, 980-8577, Japan \\ ${ }^{4}$ ISIS Facility, Rutherford Appleton Laboratory, Chilton, Didcot, OX11 OQX, UK \\ ${ }^{5}$ Department of Chemistry, Simon Fraser University, Burnaby, BC, V5A 1S6, Canada and \\ ${ }^{6}$ Ioffe Physical-Technical Institute, 26 Politekhnicheskaya, 194021 St. Petersburg, Russia
}

(Dated: June 7, 2018)

\begin{abstract}
We have used neutron scattering techniques that probe time scales from $10^{-12} \mathrm{~s}$ to $10^{-9} \mathrm{~s}$ to characterize the diffuse scattering and low-energy lattice dynamics in single crystals of the relaxor $\mathrm{PbMg}_{1 / 3} \mathrm{Nb}_{2 / 3} \mathrm{O}_{3}$ from $10 \mathrm{~K}$ to $900 \mathrm{~K}$. Our study extends far below $T_{c}=213 \mathrm{~K}$, where long-range ferroelectric correlations have been reported under field-cooled conditions, and well above the nominal Burns temperature $T_{d} \approx 620 \mathrm{~K}$, where optical measurements suggest the development of short-range polar correlations known as "polar nanoregions" (PNR). We observed two distinct types of diffuse scattering. The first is weak, relatively temperature independent, persists to at least $900 \mathrm{~K}$, and forms bow-tie-shaped patterns in reciprocal space centered on (h00) Bragg peaks. We associate this primarily with chemical short-range order. The second is strong, temperature dependent, and forms butterfly-shaped patterns centered on $(h 00)$ Bragg peaks. This diffuse scattering has been attributed to the PNR because it responds to an electric field and vanishes near $T_{d} \approx 620 \mathrm{~K}$ when measured with thermal neutrons. Surprisingly, it vanishes at $420 \mathrm{~K}$ when measured with cold neutrons, which provide $\sim 4$ times superior energy resolution. That this onset temperature depends so strongly on the instrumental energy resolution indicates that the diffuse scattering has a quasielastic character and demands a reassessment of the Burns temperature $T_{d}$. Neutron backscattering measurements made with 300 times better energy resolution confirm the onset temperature of $420 \pm 20 \mathrm{~K}$. The energy width of the diffuse scattering is resolution limited, indicating that the PNR are static on timescales of at least $2 \mathrm{~ns}$ between $420 \mathrm{~K}$ and $10 \mathrm{~K}$. Transverse acoustic (TA) phonon lifetimes, which are known to decrease dramatically for wave vectors $q \approx 0.2 \AA^{-1}$ and $T_{c}<T<T_{d}$, are temperature independent up to $900 \mathrm{~K}$ for $q$ close to the zone center. This motivates a physical picture in which sufficiently long-wavelength TA phonons average over the PNR; only those TA phonons having wavelengths comparable to the size of the PNR are affected. Finally, the PMN lattice constant changes by less than $0.001 \AA$ below $300 \mathrm{~K}$, but expands rapidly at a rate of $2.5 \times 10^{-5} 1 / \mathrm{K}$ at high temperature. These disparate regimes of low and high thermal expansion bracket the revised value of $T_{d}$, which suggests the anomalous thermal expansion results from the condensation of static PNR.
\end{abstract}

PACS numbers: 77.84.Dy, 77.65.-j, 77.80.Bh

\section{INTRODUCTION}

The concept of nanometer-scale regions of polarization, randomly embedded within a non-polar cubic matrix, has become central to attempts to explain the remarkable physical properties of relaxors such as $\mathrm{PbMg}_{1 / 3} \mathrm{Nb}_{2 / 3} \mathrm{O}_{3}$ $(\mathrm{PMN})$ and $\mathrm{PbZn}_{1 / 3} \mathrm{Nb}_{2 / 3} \mathrm{O}_{3}(\mathrm{PZN}) \stackrel{1,2,3,4}{\underline{4}}$ The existence of these so-called "polar nanoregions" (PNR) was first inferred from the optic index of refraction studies of Burns and Dacol on PMN, PZN, and other related systems,,$\underline{5}$ and later confirmed using many different experimental techniques including x-ray and neutron diffraction, $, \frac{6,8,9}{2}{ }^{207} \mathrm{~Pb} \mathrm{NMR}, \frac{10}{,}$ and piezoresponse force microscopy: 11 Early small-angle $x$-ray scattering and neutron pair distribution function (PDF) measurements on PMN by Egami et al. cast doubt on the nano-domain model of relaxors $\frac{12}{2}$ However, the recent PDF analysis of Jeong et al., which shows the formation of polar ionic shifts in PMN below $\approx 650 \mathrm{~K}$, and which occupy only one third of the total sample volume at low tempera- tures, provides convincing support for the existence of PNR $\stackrel{13}{=}$ Neutron inelastic scattering data published by Naberezhnov et al. on PMN offered the first dynamical evidence of PNR in the form of a prominent broadening of the transverse acoustic (TA) mode that coincides with the onset of strong diffuse scattering at $650 \mathrm{~K}, 14,15$ roughly the same temperature $(\approx 620 \mathrm{~K})$ as that reported by Burns and Dacol in their optical study of PMN. This temperature, commonly known as the Burns temperature, and denoted by $T_{d}$ in the original paper, is widely viewed as that at which static PNR first appear. Likely condensing from a soft TO mode, distinctive butterflyshaped and ellipsoidal diffuse scattering intensity contours centered on $(h 00)$ and $(h h 0)$ Bragg peaks, respectively, are seen below $T_{d}$ in both PMN16,17,18 and PZN $\underline{19}$ Similar diffuse scattering patterns were subsequently observed in solid solutions of PMN and PZN with $\mathrm{PbTiO}_{3}$ (PMN- $x$ PT and PZN- $x$ PT); however these patterns appear only on the Ti-poor (relaxor) side of the well-known morphotropic phase boundary (MPB) 20,21 The polar nature of the strong diffuse scattering, and thus its associ- 


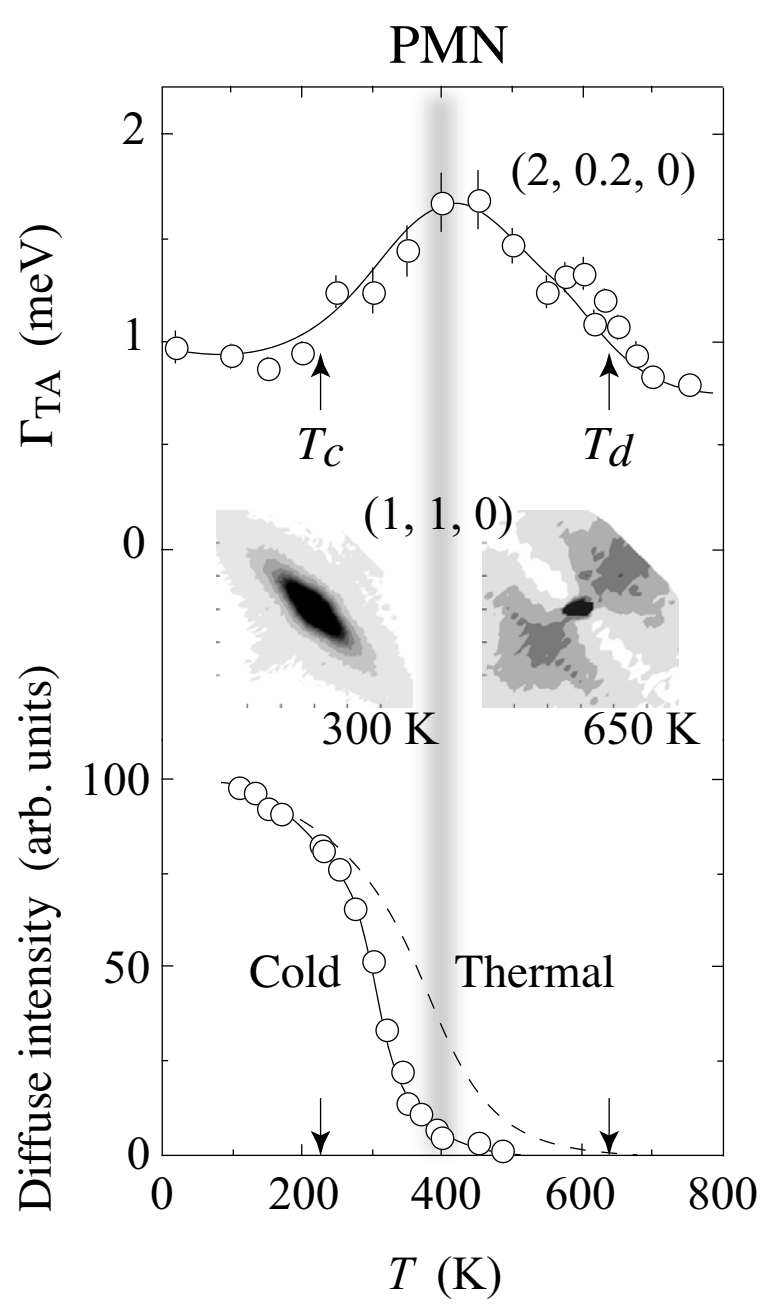

FIG. 1: Top - temperature dependence of the TA phonon energy width $\Gamma_{T A}$ measured with thermal neutrons by Wakimoto et $a l^{27}$ Middle - diffuse scattering intensity contours measured at $(110)$ below $(300 \mathrm{~K})$ and just above $(650 \mathrm{~K})$ the Burns temperature $T_{d}$ using cold neutrons by Hiraka et al. ${ }^{28}$ Bottom - diffuse scattering temperature dependence measured with cold neutrons (open circles) and thermal neutrons (dashed line) by Hiraka et al. ${ }^{28}$

ation with the formation of PNR, was unambiguously established by several electric field studies of $\mathrm{PMN}^{22,23}$ and PZN-8\%PT, $, 29,24,25,26$ all of which showed dramatic changes in the shape and intensity of the diffuse scattering as a function of field strength and field orientation. The Burns temperature $T_{d}$ thus represents what is arguably the most important temperature scale in relaxors and is several hundred degrees Kelvin higher than the critical temperature $T_{c}$ (which for PMN is $\approx 210 \mathrm{~K}$, but defined only in non-zero electric field).

Recent studies of the neutron diffuse scattering in PMN by Stock et al.,$\frac{29}{n}$ and especially those by Hlinka et $a l .30$ and Gvasaliya et al. $, 31,32$ have proven to be extremely important because they demonstrated the utility of cold neutron spectroscopy to the study of relax- ors. Generally speaking cold neutrons are ill-suited to lattice dynamical studies because the longer wavelengths necessarily limit their use to the study of comparatively fewer (lower- $Q$ ) Brillouin zones. On the other hand cold neutron spectrometers provide significantly better energy $(\hbar \omega)$ and wave vector $(q)$ resolution than do their thermal neutron counterparts. In addition, both the (110) and the (100) Brillouin zones of PMN are accessible using cold neutrons with wavelengths $\lambda \approx 4.26 \AA$. The combined cold and thermal neutron study of PMN by Hiraka et al. exploited this fact and uncovered several major new findings, two of which are summarized in Fig. 1 ${ }^{28}$ The first finding is that the temperature at which the strong diffuse scattering vanishes depends on whether the measurement is made with cold or thermal neutrons, i. e. the value of $T_{d}$ depends on the energy resolution of the spectrometer. This fact, which is illustrated in the bottom panel of Fig. [1, indicates that the diffuse scattering in PMN, and most likely other relaxors, contains a substantial quasielastic component. However, a consensus on this issue is lacking; while the finding of Hiraka et $a l$. is consistent with the study of Gvasaliya et al. $\frac{31,32}{3}$ it contradicts that of Hlinka et al $\stackrel{30,33}{=}$ A second major finding is displayed in the middle panel of Fig. 11 where intensity contours of the diffuse scattering measured with cold neutrons around (110) are shown to exhibit markedly different reciprocal space geometries at $300 \mathrm{~K}$ and $650 \mathrm{~K}$. These data unambiguously demonstrate the presence of two distinct types of diffuse scattering in PMN. As the PNR are absent at $650 \mathrm{~K} \geq T_{d}$, the physical origin of the bow-tie-shaped diffuse scattering cross section is believed to come primarily from the underlying chemical short-range order. We shall refer to this as CSRO, although another commonly-used term for this is compositionally/chemically ordered regions (COR) $\underline{\underline{34}}$

An intriguing perspective on the Burns temperature in PMN is provided in the top panel of Fig. 11 where the TA phonon energy width $\Gamma_{T A}$, which is inversely proportional to the phonon lifetime, is plotted as a function of temperature for PMN at the scattering vector $\vec{Q}=(2,0.2,0)$. These data were measured with thermal neutrons by Wakimoto et al. $\stackrel{27}{\underline{2}}$ and are consistent with those of Naberezhnov et al. in that the TA mode begins to broaden at $T_{d} \approx 620 \mathrm{~K}$, the same temperature where the strong diffuse scattering first appears. These data also show that the TA broadening achieves a maximum value (minimum lifetime) near $420 \mathrm{~K}$, which coincides with the value of $T_{d}$ measured with cold neutrons. These data raise the question of how to interpret the Burns temperature $T_{d}$ properly in that they paint a picture, markedly different from that currently held, in which the PNR are dynamic below $\sim 650 \mathrm{~K}$ and condense into static entities only at a much lower temperature of $420 \mathrm{~K}$.

The goal of our study then is to determine the intrinsic value of the Burns temperature $T_{d}$ and to clarify its relationship to the diffuse scattering, lattice dynamics, and structure in PMN. To this end we have carried out extensive measurements of the neutron diffuse scattering 
cross section from $10 \mathrm{~K}$ to $900 \mathrm{~K}$, far below $T_{c}$ and well above the nominal value of $T_{d}$, that probe timescales from $10^{-12} \mathrm{~s}$ to $10^{-9} \mathrm{~s}$. We find that a 300 -fold improvement in energy resolution over that used by Hiraka et al. 28 obtained using neutron backscattering techniques reproduces the same onset temperature for the diffuse scattering; hence the intrinsic Burns temperature $T_{d}$ for PMN is $420 \mathrm{~K}$. At the same time an enormous change in the thermal expansion is observed near $420 \mathrm{~K}$, which is indistinguishable from zero at low temperature. Given the revised value of $T_{d}$, this result implies the existence of a direct influence of the PNR on the intrinsic structural properties of PMN.

We also present new data on the effects of the PNR on the lattice dynamics through measurements of the temperature and wave vector dependence of the longwavelength TA phonon energy width $\Gamma_{T A}$ measured near (110) from $25 \mathrm{~K}$ to $900 \mathrm{~K}$. We find that TA modes with reduced wave vectors $q \ll 0.2 \AA^{-1}$ exhibit the same energy width at all temperature whereas those with $q \approx$ $0.2 \AA^{-1}$ exhibit a strongly temperature-dependent broadening similar to that shown in the top panel of Fig 1 This behavior contrasts with that observed in thermal neutron studies of the TO mode, which exhibits a broadening for all $q \leq 0.2 \AA^{-1}$. Previous neutron scattering work on PMN-60\% PT by Stock et al. $\frac{35}{2}$ a material in which there is no strong, temperature dependent diffuse scattering, and thus no polar nanoregions, found no evidence of any TA phonon broadening. In this context, our data lend extremely strong support to the PNR model: the lifetimes of TA modes with wavelengths comparable in size to the PNR are strongly diminished by the PNR, whereas long-wavelength (low $q$ ) TA phonons simply average over the PNR and are unaffected.

\section{EXPERIMENTAL DETAILS}

The neutron scattering data presented here were obtained using the BT9 thermal neutron triple-axis spectrometer, the SPINS cold neutron triple-axis spectrometer, and the cold neutron High Flux Backscattering Spectrometer (HFBS), all of which are located at the NIST Center for Neutron Research (NCNR). On BT9, measurements of the phonons and diffuse scattering were made at a fixed final (thermal) neutron energy $E_{f}=14.7 \mathrm{meV}(\lambda=2.36 \AA)$ using the (002) Bragg reflection of highly-oriented pyrolytic graphite (HOPG) crystals to monochromate and analyze the incident and scattered neutron beams, respectively. Horizontal beam collimations were $40^{\prime}-47^{\prime}-\mathrm{S}-40^{\prime}-80^{\prime}(\mathrm{S}=$ sample). A special, non-standard high $q$-resolution configuration was employed to measure the thermal expansion in which the (004) Bragg reflection from a perfect Ge crystal was used as analyzer and horizontal beam collimations were tightened to $15^{\prime}-47^{\prime}-\mathrm{S}-20^{\prime}-40^{\prime}$. The choice of Ge was motivated by the close matching between the PMN (022) (1.431 $\AA$ ) and Ge (004) (1.414 $\AA$ ) $d$-spacings, which provides a significant improvement in the instrumental $q$-resolution 36 On SPINS, which sits on the cold neutron guide NG5, the phonon and diffuse scattering measurements were made at a fixed final neutron energy $E_{f}=4.5 \mathrm{meV}(\lambda=4.264 \AA)$ also using the (002) Bragg reflection of HOPG crystals as monochromator and analyzer. A liquid-nitrogen cooled Be filter was located after the sample to remove higher order neutron wavelengths from the scattered beam, and horizontal beam collimations were set to guide- $80^{\prime}-\mathrm{S}-80^{\prime}-80^{\prime}$. The resultant elastic $(\hbar \omega=0)$ energy resolution for the SPINS measurements was $\delta E=0.12 \mathrm{meV}$ half-width at half-maximum (HWHM).

The High-Flux Backscattering Spectrometer was used to look for dynamics that might be associated with the strong diffuse scattering below $T_{d}$. This instrument uses a mechanically-driven $\mathrm{Si}(111)$ monochromator to Doppler shift the energies of incident neutrons over a narrow range centered about $2.08 \mathrm{meV}$. Neutrons are backscattered from the monochromator and proceed towards the sample where they are scattered into a $12 \mathrm{~m}^{2}$ array of $\mathrm{Si}$ (111) crystals that serve as analyzer. These neutrons are then backscattered a second time by the analyzer, which selects the final neutron energy $E_{f}=$ $2.08 \mathrm{meV}$, into a series of detectors positioned about the sample. The effective angular acceptance of each detector is $\approx 15^{\circ}$. The HFBS instrument is described in further detail elsewhere 37 The elastic energy resolution for the HFBS measurements described here was $\delta E=0.4 \mu \mathrm{eV}$ (HWHM).

Two high-quality single crystals of PMN, labeled PMN \#4 and PMN \#5, were used in this study; both were grown using a top-seeded solution growth technique $\stackrel{38}{=}$ The crystal growth conditions were determined from the pseudo-binary phase diagram established for $\mathrm{PMN}$ and $\mathrm{PbO}$. The PMN \#4 and \#5 crystals weigh $2.7 \mathrm{~g}\left(0.33 \mathrm{~cm}^{3}\right)$ and $4.8 \mathrm{~g}\left(0.59 \mathrm{~cm}^{3}\right)$, respectively. At $300 \mathrm{~K}$ the mosaic of each crystal measured at (220) is less than $0.04^{\circ}$ full-width at half-maximum (FWHM). Loss of $\mathrm{PbO}$, the formation of a pyrochlore phase, and the reduction of $\mathrm{Nb}^{5+}$ are known to occur in PMN single crystals when subjected to high temperatures under vacuum for extended periods of time. This process results in a dramatic blackening of the crystal, which is normally of a transparent gold/amber color. While dielectric measurements on such darkened crystals reportedly show little difference from those on unheated samples, $\stackrel{39}{\underline{3}}$ our measurements reveal a diminishment of the diffuse scattering intensity after sustained and repeated heating. Therefore experiments on the larger PMN crystal \#5 were limited to $600 \mathrm{~K}$ or less, while PMN crystal \#4 was used to obtain data above $600 \mathrm{~K}$.

Both samples were mounted with an [001] axis oriented vertically, giving access to reflections of the form $(h k 0)$. For the high temperature experiments, PMN crystal \#4 was wrapped in quartz wool, mounted in a niobium holder secured by tungsten wire, and then loaded into a water-cooled furnace capable of reaching temperatures 


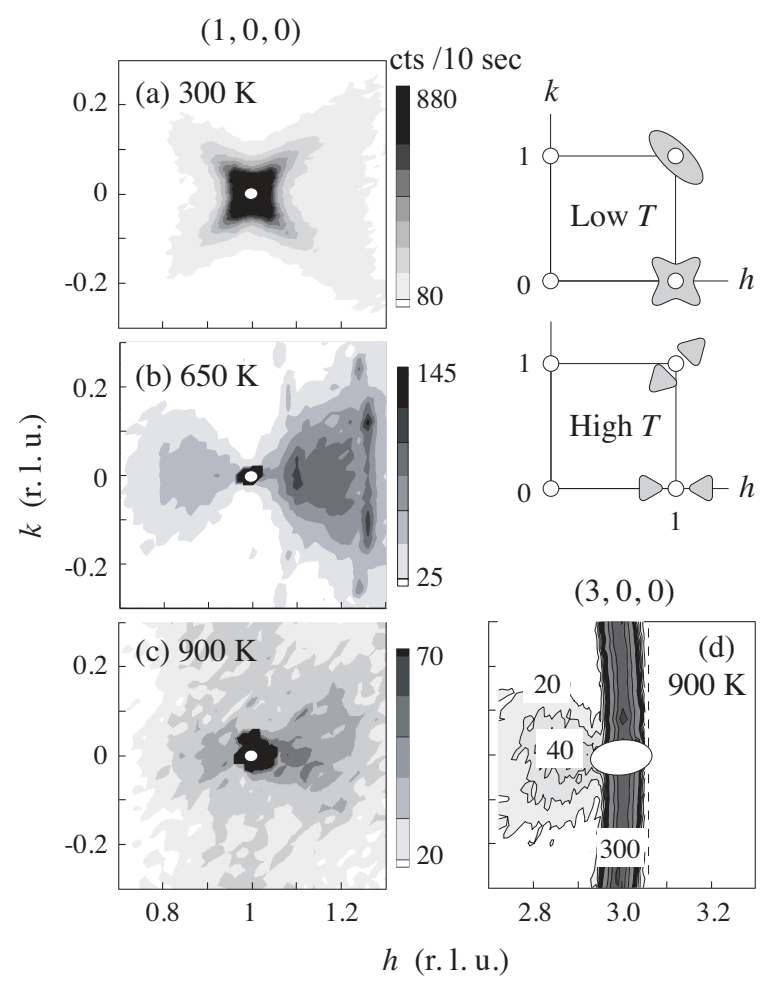

FIG. 2: Diffuse scattering intensity contours in PMN measured on SPINS near (100) are shown using a linear gray scale at (a) $300 \mathrm{~K}$, (b) $650 \mathrm{~K}$, and (c) $900 \mathrm{~K}$. Diffuse scattering intensity contours measured on BT9 at $900 \mathrm{~K}$ near (300) using thermal neutrons are shown in (d). The data shown in panels (a) and (b) are from Hiraka et al. ${ }^{28}$ Schematic diagrams of the diffuse scattering intensity contours below (Low- $T$ ) and at $T_{d}$ (High- $T$ ) are shown in the upper right.

from $300 \mathrm{~K}$ to $1800 \mathrm{~K}$. PMN crystal \#5 was mounted onto an aluminum sample holder assembly placed inside an aluminum sample can, and then loaded inside the vacuum space of a closed-cycle ${ }^{4} \mathrm{He}$ refrigerator that provides access to temperatures from $10 \mathrm{~K}$ to $700 \mathrm{~K}$. Each sample has a cubic lattice spacing of $a=4.05 \AA$ at $300 \mathrm{~K}$; thus $1 \mathrm{rlu}$ (reciprocal lattice unit) equals $2 \pi / a=1.55 \AA^{-1}$.

\section{ORIGINS OF THE DIFFUSE SCATTERING: PNR VERSUS CSRO}

The diffuse scattering in PMN crystal \#5 was studied by Hiraka et al. $\frac{28}{}$ at and below the nominal Burns temperature $T_{d}$ with good energy resolution $(\delta E \approx 0.12 \mathrm{meV}$ HWHM) using cold neutrons. Data were measured in the (100) and (110) Brillouin zones and are represented schematically in the upper right portion of Fig. 2. The horizontal and vertical axes in this figure correspond to the components of the scattering vector $\vec{Q}=(h k 0)$, which are measured in reciprocal lattice units (rlu). The "Low- $T$ " $\left(T<T_{d}\right)$ regime is dominated by contributions from the PNR, where the diffuse scattering intensity con- tours near (100) resemble a butterfly and those near (110) resemble an ellipse for which the long axis is oriented perpendicular to $\vec{Q}$. This is shown in Fig. 2(a) where diffuse scattering data near (100) at $300 \mathrm{~K}$ reveal an intense butterfly-shaped pattern $\stackrel{28}{\underline{ }}$ We note here that we see no evidence of any "transverse component" to the diffuse scattering in any of our PMN crystals like that reported previously by Vakhrushev et al. $\stackrel{41}{*}$ We assume that this component was due to the crystal imperfections mentioned by these authors in their PMN sample, which gave rise to a powder ring in their data at (100).

The same butterfly/ellipsoidal diffuse scattering geometry was shown to persist in single crystals of PMN$x \mathrm{PT}$ and $\mathrm{PZN}-x \mathrm{PT}$ in studies by, respectively, Matsuura et al. and $\mathrm{Xu}$ et al. for compositions spanning the Tipoor (relaxor) side of the morphotropic phase boundary (MPB) 20.21 These results also completely refute those of La-Orauttapong et al. who reported that the orientation of the strong diffuse scattering varies with Ti content in PZN- $x \mathrm{PT}$ and concluded that the PNR orientation changes with doping $\underline{\underline{42,43}}$ For Ti-rich (tetragonal) PMN- $x$ PT compositions just beyond the MPB, Matsuura et al. found that the strong, temperature dependent diffuse scattering vanishes and is replaced by critical scattering. ${ }^{20}$ Matsuura et al. also found that the $q$ integrated diffuse scattering intensity increases with $\mathrm{Ti}$ content on the Ti-poor side of the MPB, peaks near the MPB, then drops dramatically on crossing the MPB. This finding is significant because it suggests that an intriguing and direct correlation exists between the PNR and the piezoelectric coefficient $d_{33}$, which exhibits the same dependence on $\mathrm{Ti}$ content $\underline{\underline{2}}$ A model based on pancake-shaped, ferroelectric domains has been used successfully to fit the three-dimensional diffuse scattering

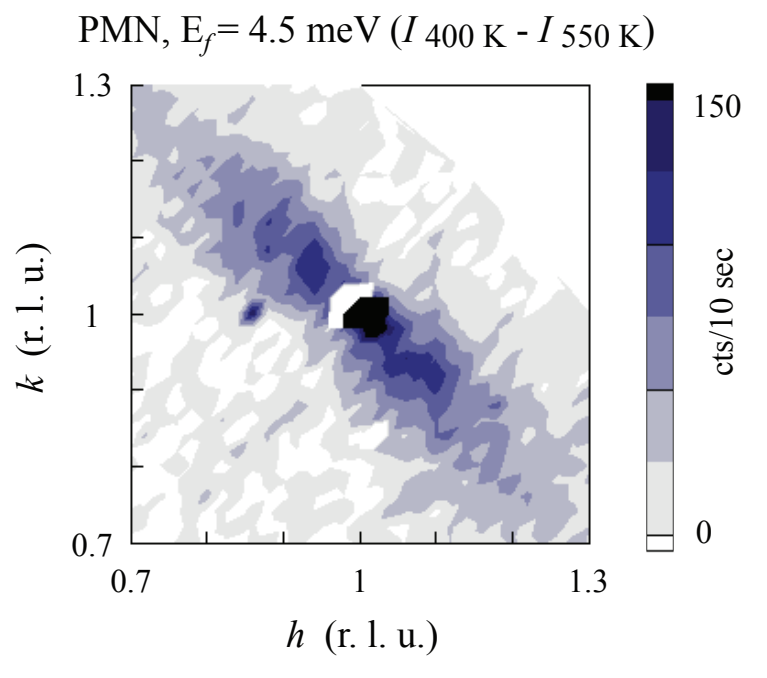

FIG. 3: Contour plot of the difference in diffuse scattering intensity near (110) measured at $550 \mathrm{~K}$ and $400 \mathrm{~K}$. The result is the characteristic figure " 8 " pattern, observed previously by Vakhrushev et al. ${ }^{40}$ 
distributions measured in PZN- $x$ PT with high-energy $\mathrm{x}$ rays.21,44,45 A similar type of real-space structure has been proposed to explain the diffuse scattering in the relaxor $\mathrm{KLi}_{x} \mathrm{Ta}_{1-x} \mathrm{O}_{3} \stackrel{46}{ }$ On the other hand, alternative models explaining the same diffuse scattering distributions have also been proposed. 47

In the "High- $T$ " regime $\left(T \geq T_{d}\right)$ there are no PNR, and the associated butterfly-shaped diffuse scattering is no longer present. The weak diffuse scattering that remains is thus argued to originate primarily from the underlying chemical short range order (CSRO), which reflects weak correlations between the $\mathrm{Mg}^{2+}$ and $\mathrm{Nb}^{5+}$ cations on the $B$-site of the perovskite $A B \mathrm{O}_{3}$ structure. In this regime the shapes of the diffuse scattering contours are radically different, resembling a bow-tie in both $\vec{Q}=(h 00)$ and $\vec{Q}=(h h 0)$ Brillouin zones in which the diffuse scattering extends mainly parallel to $\vec{Q}$. The only difference between the contours near (100) and (110) appears to be in the orientation of the triangular regions of diffuse scattering, which point in towards (100), but away from (110). Data taken near (100) at $650 \mathrm{~K}$ are displayed in Fig. 2(b) ${ }^{28}$ At this temperature the diffuse scattering intensities, shown using a linear gray scale, are much weaker than those of the butterfly pattern at $300 \mathrm{~K}$. We further note that the intensities increase (become darker) from left to right in panels (b) and (c), which corresponds to increasing $Q$. Given the $(\vec{Q} \cdot \vec{u})^{2}$ dependence of the neutron diffuse scattering cross section, this intensity signature implies the presence of some short-range, correlated displacements $\vec{u}$ since otherwise, if $u=0$, there would be no $Q$-dependence. Thus the weak diffuse scattering is not solely due to CSRO.

In Fig. 3 we plot the difference between the diffuse scattering intensities measured near $\vec{Q}=(110)$ at $400 \mathrm{~K}$ and $550 \mathrm{~K}$. The resulting contours approximately reproduce the "figure 8" pattern observed previously by Vakhrushev et al. $\stackrel{40}{,}$, and indicates that the ellipsoidal diffuse scattering has a strong temperature dependence. By contrast, the bow-tie-shaped diffuse scattering is effectively subtracted out in this analysis, which confirms that it has little to no temperature dependence. Thus the hightemperature diffuse scattering is not associated with the Burns temperature $T_{d}$ or with the formation of longrange polar correlations below $T_{c}$. We note that such a strictly incoherent treatment of the high-temperature (CSRO) and low-temperature (PNR) components of the total diffuse scattering, as described here, ignores the inevitable cross terms that must exist between them. On the other hand, if the high-temperature scattering does arise primarily from CSRO, then it should be largely independent of the ionic displacements that give rise to the butterfly-shaped diffuse scattering (PNR) below $T_{d}$. The relative weakness of the high-temperature diffuse scattering compared to that at low temperatures also suggests such cross terms should be weak, and this appears to be supported by the simple subtraction analysis presented in Fig. 3 in that one effectively recovers the ellipsoidal (not bow-tie) intensity contours. For this reason we be-
PMN 14.7Ef 40'-46'-40'-80'

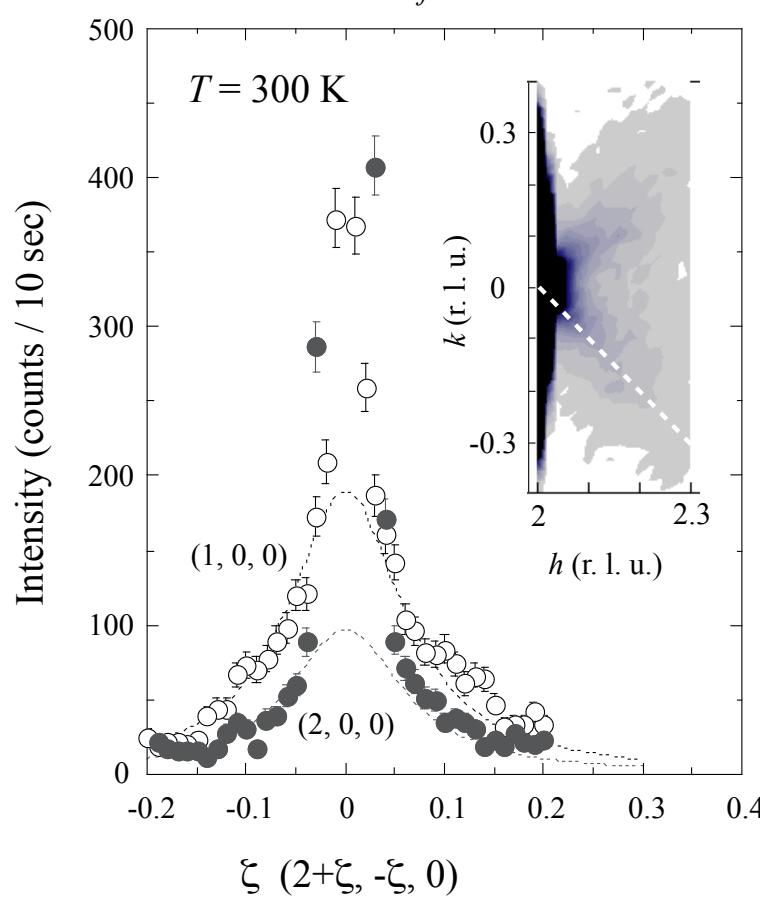

FIG. 4: Diffuse scattering intensity measured on BT9 at $300 \mathrm{~K}$ as a function of reduced momentum transfer relative to (100) (open circles) and (200) (solid circles). The dotted lines isolate the diffuse scattering component from the Bragg peak. The inset shows the orientation of the scan relative to the familiar butterfly pattern measured near (200) at $100 \mathrm{~K}$.

lieve it is a reasonable first approximation to treat the two diffuse scattering components as being nearly independent.

We extended the diffuse scattering measurements to temperatures well above $T_{d}$ using PMN crystal \#4, which we reserved for very high temperature experiments. Previous data taken on PMN and PZN crystals heated to $1000 \mathrm{~K}$ revealed significant evidence of sample decomposition, so we limited our measurements to $900 \mathrm{~K}$. Diffuse scattering intensity contours at $900 \mathrm{~K}$ for the (100) and the (300) Brillouin zones are presented in Fig. 2(c) and 2](d), respectively. Although the diffuse scattering near (100) is quite weak, it is consistent with the bow-tie geometry observed in Fig. 2(b) at $650 \mathrm{~K}$. We exploited the $Q^{2}$ dependence of the neutron diffuse scattering cross section to obtain higher intensity by using thermal neutrons to access (300). The (300) diffuse scattering intensity contours are shown in Fig. 2(d), where the bow-tie pattern observed at $650 \mathrm{~K}$ is still present at $900 \mathrm{~K}$. The contours are truncated for $h>3.02 \mathrm{rlu}$ because of a mechanical limit on the maximum scattering angle available on BT9, but the triangular region on the low- $Q$ side of (300) is clearly evident. That the bow-tie-shaped diffuse scattering persists to such high temperature provides what is perhaps the most convincing evidence that it arises mainly from CSRO. 


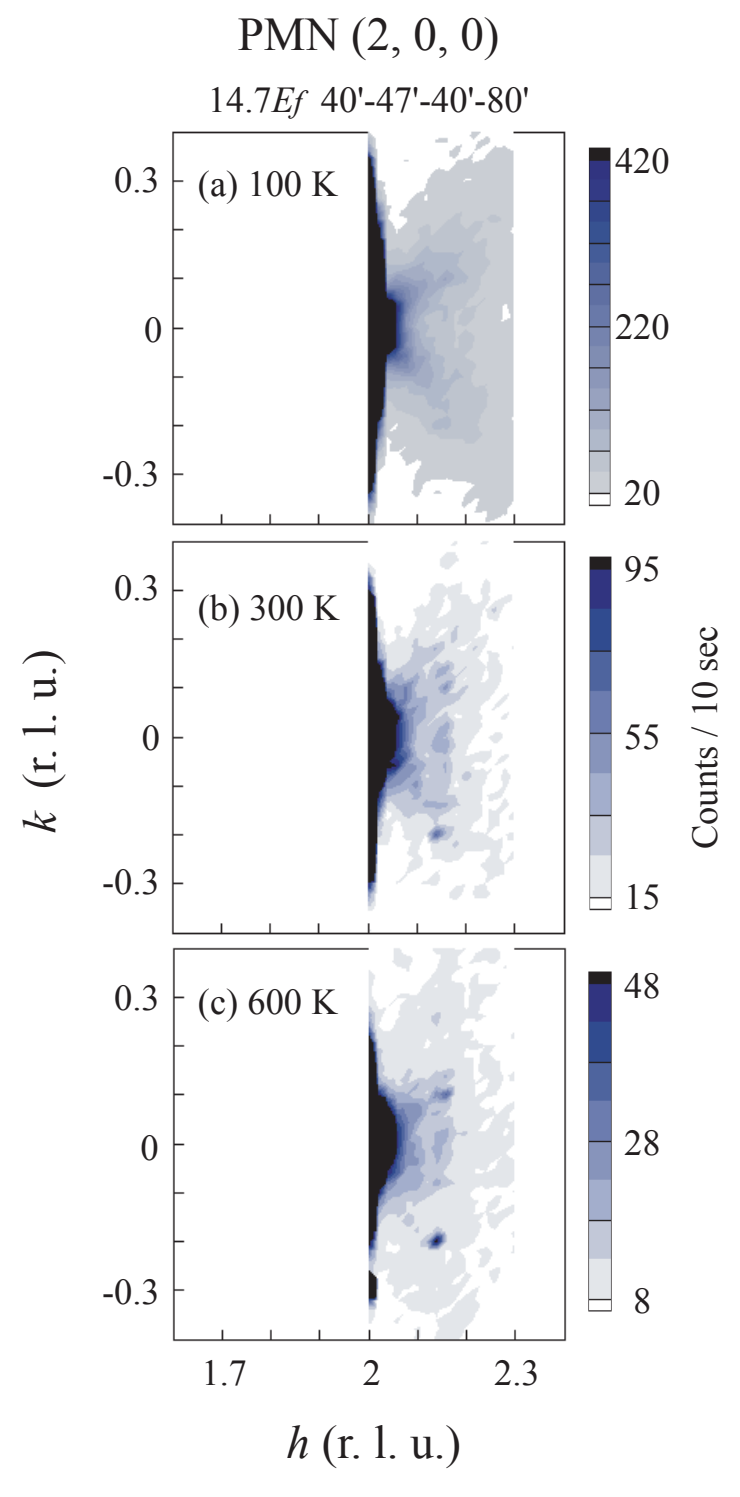

FIG. 5: Diffuse scattering contours measured on BT9 near (200) are shown at (a) $100 \mathrm{~K}$, (b) $300 \mathrm{~K}$, and (c) $600 \mathrm{~K}$.

During the course of our measurements we noticed that the diffuse scattering in PMN crystal \#4 had diminished and was noticeably weaker than that in PMN crystal \#5, which we had never exposed to temperatures above $650 \mathrm{~K}$. We also observed a broad ring of scattering passing directly through $\vec{Q}=(300)$, which is shown in Fig. 2(d). This feature was never observed prior to heating this crystal to $900 \mathrm{~K}$. Given the length of time spent at high temperatures, this feature most likely corresponds to a powder ring arising from partially decomposed regions of PMN crystal \#4, which had turned entirely black after exposure to high temperatures. These regions do not affect any other data presented in this paper because the $900 \mathrm{~K}$ measurements on PMN crystal \#4 were the last ones performed on this sample. Therefore the powder ring appears only in Fig. 2(d).

It is instructive to compare these results with those on PMN-60\%PT, a composition that lies well beyond the morphotropic phase boundary and undergoes a firstorder ferroelectric transition from a cubic to a tetragonal phase near $540 \mathrm{~K}$. An extensive study of this material using neutron and high-energy x-ray scattering methods found no sign of the strong, butterfly-shaped diffuse scattering at low temperatures 35 This result lends further support to our association of the strong, temperature dependent diffuse scattering with the PNR, which are absent in PMN-60\%PT. Neutron measurements on PMN-60\%PT do, however, reveal the presence of bow-tie shaped diffuse scattering intensity contours at all temperatures studied, which supports the identification of such diffuse scattering with chemical short-range order between cations on the $B$ site of the PMN perovskite $A B \mathrm{O}_{3}$ structure. This picture is supported by theoreti-

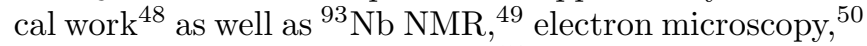
and polarized Raman scattering ${ }^{51}$ measurements. All of these studies suggest that there is no temperature dependence to the bow-tie shaped diffuse scattering below $\approx 1000 \mathrm{~K}$, which is consistent with our results on PMN over the extended temperature range.

During our study of PMN we discovered that the diffuse scattering near (200) is not as weak as previously believed $\underline{17,40,42,52}$ To confirm this finding, we made detailed measurements of the diffuse scattering intensity near (200) at $300 \mathrm{~K}$ and $100 \mathrm{~K}$ along a trajectory in reciprocal space that follows one wing of the butterfly intensity contour; this is shown by the dashed line in the inset to Fig. (4) The results of the $300 \mathrm{~K}$ scan are compared to an identical scan measured in the (100) zone, both of which are shown in Fig. 4, These data demonstrate that the (100) diffuse scattering cross section, represented by the dotted lines passing through the open circles, is substantially larger than that at (200), designated by the solid circles. This result supports the model of Hirota et al. in which the (unexpectedly) weak (200) diffuse scattering cross section observed in PMN and other relaxors can be explained by the presence of a uniform shift or displacement of the PNR relative to the non-polar cubic matrix along the direction of the local

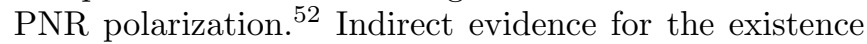
of this shift has been obtained from neutron scattering measurements of the anisotropic response of the diffuse scattering in PZN-8\%PT to an electric field applied along the [001] direction ${ }^{24}$

Fig. 5 shows diffuse scattering intensity contours measured on BT9 at $100 \mathrm{~K}, 300 \mathrm{~K}$, and $600 \mathrm{~K}$ near(200); these data illustrate that the (200) diffuse scattering intensity follows the same temperature dependence as that measured in other Brillouin zones, where the diffuse scattering is much stronger. As the temperature is raised the diffuse scattering intensity decreases in the same manner as that previously measured and observed in the (100), (110), and (300) Brillouin zones. This proves that the diffuse scattering measured at (200) has the same origin as 
PMN, T=300 K, DCS (Ei=2.70 meV)

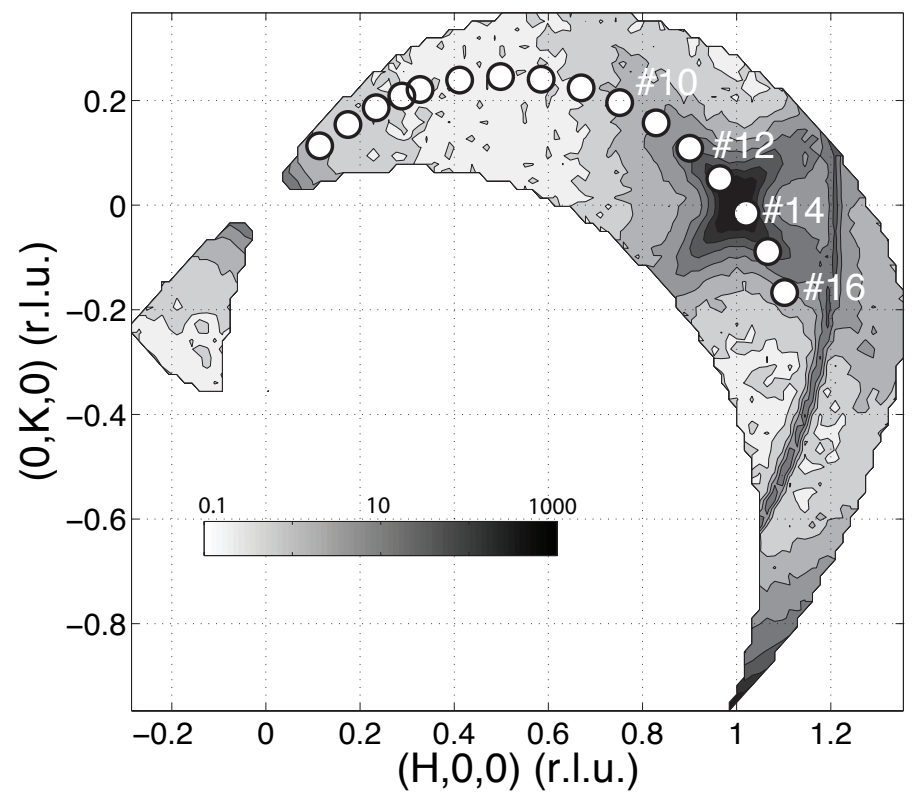

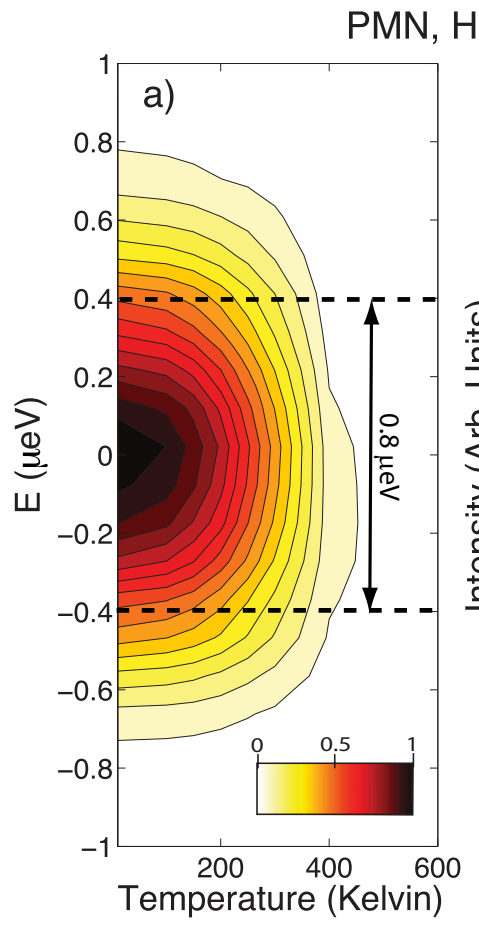

PMN, HFBS, Q=(100)

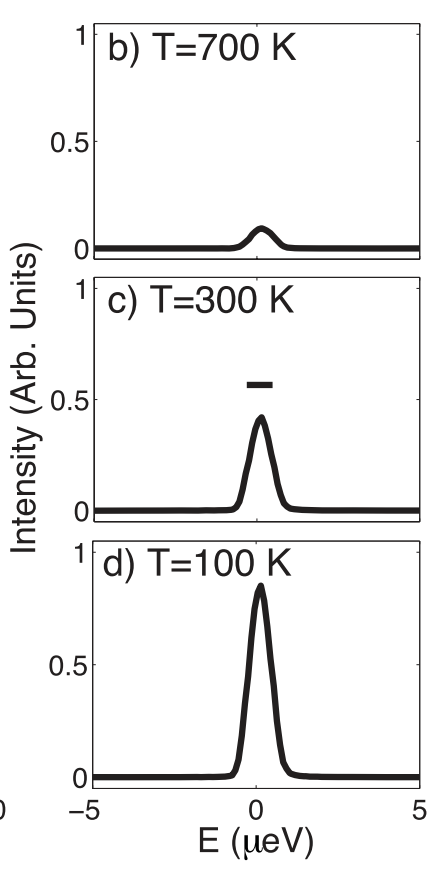

FIG. 6: Diffuse scattering intensity contours in PMN measured on the NCNR Disk Chopper Spectrometer (DCS) near (100) are plotted on a logarithmic gray scale at $300 \mathrm{~K}$. Open circles represent the reciprocal space locations of the detectors of the High Flux Backscattering Spectrometer relative to the butterfly-shaped intensity contours. Each open circle is a separate detector, and the detector numbers are indicated. These DCS data are taken from Xu et al. ${ }^{18}$

that in other zones, i. e. that it is associated with the formation of PNR. At $600 \mathrm{~K}$ in panel (c) one can already see the emergence of the bow-tie-shaped diffuse scattering that is otherwise obscured by the stronger PNR-related diffuse scattering at lower temperatures. These data are important because they support the mode-coupling analysis of Stock et al. ${ }^{29}$, which assumes that the diffuse scattering in PMN in the (200) and (220) Brillouin zones is much weaker than that in the (110) zone. Thus we emphasize that while the neutron diffuse scattering crosssection near (200) is not zero, it is small and consistent with previous structure factor calculations.

\section{DIFFUSE SCATTERING DYNAMICS: A REASSESSMENT OF THE BURNS TEMPERATURE}

The reciprocal space geometry of the strong diffuse scattering in PMN was first characterized using x-ray diffraction and is consistent with the neutron scattering data we have presented here ${ }^{16,17}$ The energy resolution provided by $\mathrm{x}$-ray diffraction $(\delta E \approx 1000 \mathrm{meV})$ is typically much broader than that of thermal neutrons $(\delta E \approx 1 \mathrm{meV})$; thus it was assumed that the strong diffuse x-ray scattering originated from low-energy, soft,

FIG. 7: (Color online) (a) Contour plot of the diffuse scattering intensity measured on the HFBS as a function of energy transfer and temperature. Contours are shown on a linear intensity scale; dashed lines indicate the full-width at halfmaximum (FWHM) of the peak linewidth at each temperature. Data were summed over detectors 10-16 as illustrated in Fig. 6] Panels (b), (c), and (d) show inelastic scans at $700 \mathrm{~K}$, $300 \mathrm{~K}$, and $100 \mathrm{~K}$. The horizontal bar in panel (c) represents the instrumental FWHM elastic energy resolution $(2 \delta E)$.

transverse optic (TO) phonons that were captured by the large energy resolution. ${ }^{17,53}$ However the cold neutron data of Hiraka al. provide a much narrower elastic energy resolution of $\approx 0.12 \mathrm{meV}$ HWHM and show, unambiguously, that the diffuse scattering cross section contains a component that is static on timescales of at least $\sim 6$ ps below $420 \mathrm{~K}$ as illustrated in Fig. 1. This result was subsequently confirmed on a separate PMN crystal by the neutron study of Gvasaliya et al. which employed comparable energy resolution 54 Hence the observed strong diffuse scattering cannot simply be the result of a soft, low-lying TO phonon. The TO mode must condense and/or broaden sufficiently to produce the elastic diffuse scattering cross section observed by Hiraka et al. Such a scenario is in fact suggested by the corresponding thermal neutron data taken on BT9 using a somewhat coarser energy resolution of $\approx 0.50 \mathrm{meV}$ HWHM. As shown in Fig. 1] an apparent elastic diffuse scattering cross section is observed up to temperatures as high as $650 \mathrm{~K}$.

Motivated by these results, we looked for evidence of a dynamic component of the strong diffuse scattering using the NCNR High-Flux Backscattering Spectrome- 


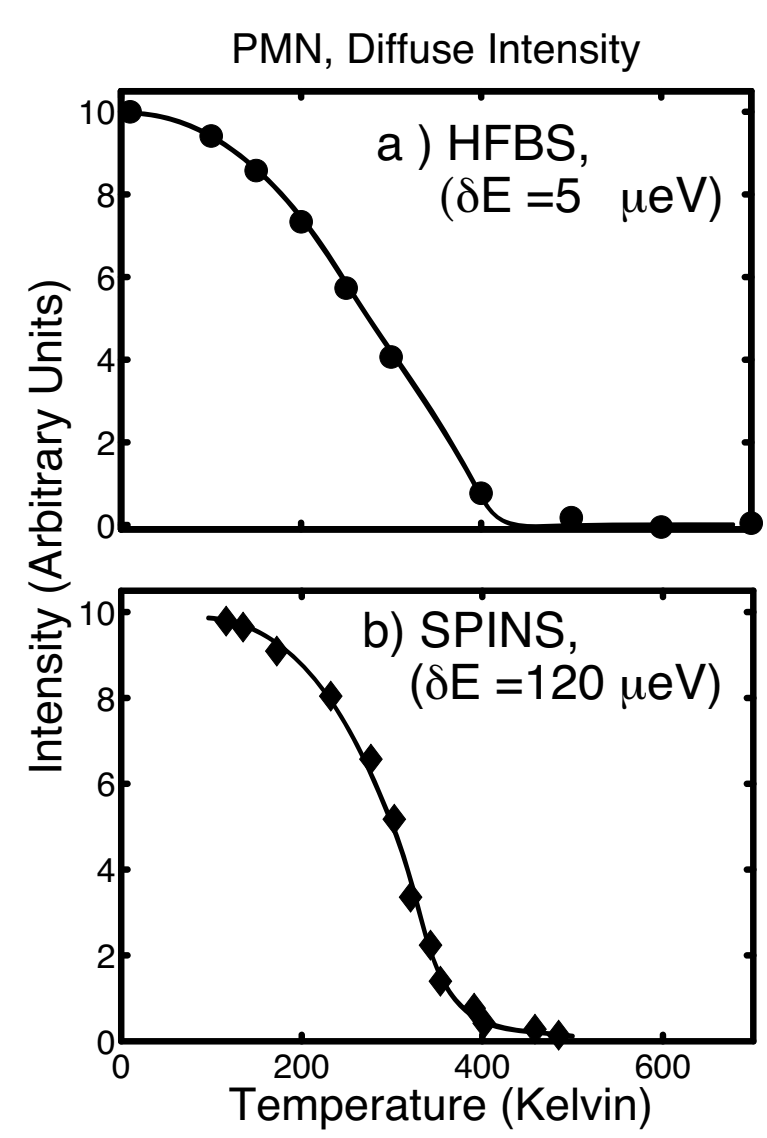

FIG. 8: (a) Temperature dependence of the diffuse scattering measured on the HFBS; data are integrated in energy from \pm $5 \mu \mathrm{eV}$ and in $Q$ over detectors 10-16 as illustrated in Fig. 6] (b) Temperature dependence of the diffuse scattering intensity measured on SPINS at $(1.05,0.95,0)$ by Hiraka et al. ${ }^{28}$ The SPINS energy resolution of $\delta E=120 \mu \mathrm{eV}$ HWHM provided the energy integration.

ter (HFBS), which provides an elastic energy resolution $\delta E=0.4 \mu \mathrm{eV}$ HWHM. We oriented PMN crystal \#4 in the $(h k 0)$ scattering plane, which is the same geometry used for the triple-axis studies discussed in the previous sections. Fig. [ displays the resulting locations of each of the 16 HFBS detectors relative to the butterfly-shaped diffuse scattering pattern at (100) measured previously using the NCNR Disk Chopper Spectrometer (DCS) $\underline{18}$ From this figure it can be seen that detectors 10 through 16 sample different parts of the wings of the strong diffuse scattering. In particular, detectors 13 and 14 lie close to the (100) Bragg peak. Because the instrumental $Q$-resolution of the HFBS is relatively poor, we checked for the presence of Bragg contamination in the integration analysis by removing contributions from detectors 13 and 14. This did not change any of the results. Moreover the PMN study by Wakimoto et al. showed the (202) Bragg peak intensity changes by less than $10 \%$ between $50 \mathrm{~K}$ and $300 \mathrm{~K} \stackrel{55}{ }$ Therefore, in the following analysis we have integrated the intensity from detectors 10 through 16.

The energy dependence of the diffuse scattering as a function of temperature is illustrated in Fig. 7 Panel (a) shows a color contour plot of the peak linewidth in energy as a function of temperature after subtraction of a high temperature background. This background was taken to be the average of the intensity measured at $600 \mathrm{~K}$ and $700 \mathrm{~K}$ because no strong diffuse scattering is observed with either cold neutron triple-axis or neutron backscattering methods at these temperatures. The distance between the dashed lines represents the measured energy width (full-width at half-maximum) as a function of temperature. Typical energy scans are displayed in panels (b), (c), and (d) at $700 \mathrm{~K}, 300 \mathrm{~K}$, and $100 \mathrm{~K}$, respectively. We see that the peak centered at $\hbar \omega=0$ is equal to the instrumental energy resolution $\delta E=0.4 \mu \mathrm{eV}$ HWHM at all temperatures. Based on this analysis, we conclude that the strong diffuse scattering is elastic on timescales of at least $\tau \approx \hbar / \delta E \sim 2$ ns. Our results are thus consistent with the neutron-spin echo study on PMN of Vakhrushev et al $\underline{41}$

Fig. 8 (a) shows the temperature dependence of the diffuse scattering intensity integrated over $\pm 5 \mu \mathrm{eV}$. These data are compared to those measured on PMN crystal \#5 using the cold neutron spectrometer SPINS, which are plotted in panel (b). That the temperature dependences from the backscattering and SPINS measurements agree within error in spite of a 300 -fold $(120 \mu \mathrm{eV} / 0.4 \mu \mathrm{eV})$ difference in energy resolution proves that static, shortrange polar order first appears at much lower temperatures than has been understood from previous data measured with much coarser energy resolution. These data thus demand a revised value for the Burns temperature: $T_{d}=420 \pm 20 \mathrm{~K}$. We mention, however, that if the diffuse scattering energy width obeys an Arrhenius or some power law form, which has been suggested for spin glasses in Ref. 56, then a more detailed analysis based on data taken closer to the onset of the diffuse scattering at $420 \mathrm{~K}$ will be required to confirm (or reject) the existence of such alternative dynamic contributions to the diffuse scattering energy width.

To gain a better understanding of the apparent quasielastic nature of the diffuse scattering, we examined the temperature dependence of the low-energy transverse acoustic (TA) modes in greater detail. In particular we focused on measurements of the temperature dependent broadening of the transverse acoustic phonon over a range of reduced wave vectors $q$ that approach the zone center $(q=0)$. These data are discussed in the following section.

\section{TA AND TO PHONONS: EFFECTS OF THE PNR}

An extensive series of inelastic measurements were made on both PMN crystals \#4 and \#5 in the (110) 
Brillouin zone using the SPINS spectrometer in an effort to map out the temperature dependence of the TA mode for reduced wave vectors $q \ll q_{w f}$ and $q \approx q_{w f}$, where $q_{w f}=0.14 \mathrm{rlu} \approx 0.2 \AA^{-1}$ is the wave vector associated with the so-called "waterfall" effect, and below which the long-wavelength, soft TO modes in PMN, PZN, and PZN-8\%PT are observed to broaden markedly at temperatures below $T_{d} \underline{27,57,58,59,60}$ This zone was chosen because the TA phonon dynamical structure factor for (110) is much larger than that for (100). The TA phonon energy lineshapes were studied at two very different values of the reduced wave vector $q$, measured relative to the (110) zone center, for temperatures between $100 \mathrm{~K}$ and 900 K. These data are presented in Fig. 9] The data shown on the left-hand side of this figure correspond to $\vec{Q}=(1.035,0.965,0)$ or $q=\sqrt{2} \cdot 0.35 \mathrm{rlu}=0.05 \mathrm{rlu}=$ $0.077 \AA^{-1}$, which is less than half of $q_{w f}$. These data show that the TA lineshape at this small wave vector remains sharp and well-defined at all temperatures and has an intrinsic energy width that is larger than the instrumental resolution (shown by the small, solid horizontal bar at $500 \mathrm{~K}$ ). By contrast, the data on the righthand side of Fig 9 correspond to $\vec{Q}=(1.1,0.9,0)$ or $q=\sqrt{2} \cdot 0.10 \mathrm{rlu}=0.14 \mathrm{rlu}=0.22 \AA^{-1}$, which is nearly equal to $q_{w f}$. In this case it is quite evident that the TA lineshape broadens dramatically, becoming increasingly ill-defined below $T_{d}$, especially at $300 \mathrm{~K}$, but then sharpens at lower temperature, e. g. at $100 \mathrm{~K}$. This behavior is the same as that observed for the soft, zone-center TO mode in PMN by Wakimoto et al. measured at (200) 27

In addition to these cold neutron measurements of the TA mode using SPINS, other measurements were made using thermal neutrons on BT9 to characterize the TO mode lineshapes for reduced wave vectors $q \approx q_{w f}$ from $300 \mathrm{~K}$ to $900 \mathrm{~K}$. In these experiments data were taken using a water-cooled furnace with PMN crystal \#4 in both the (300) and (100) Brillouin zones for $q=q_{w f}=0.14 \mathrm{rlu}$ and are shown in Fig 10, As can be seen on the lefthand side of this figure, both the TA and TO modes are well-defined at $900 \mathrm{~K}$. The scattering intensity below 1-2 meV increases sharply because of the comparatively coarser energy resolution intrinsic to BT9 $(\approx 0.5 \mathrm{meV}$ HWHM), which uses thermal neutrons, compared to that on SPINS. Even so, the TA mode is easily seen at both $900 \mathrm{~K}$ and $700 \mathrm{~K}$. However at $500 \mathrm{~K}$ the TA mode is less well-defined. This occurs in part because the mode has broadened, but also because the low-energy scattering has increased, which results from the onset of the strong diffuse scattering due to the PNR. At $300 \mathrm{~K}$ the TA mode is nearly indistinguishable from the sloping background, and the TO mode has become significantly damped. One also sees a gradual softening of the TO mode from $900 \mathrm{~K}$ down to $300 \mathrm{~K}$ that is of order $1-2 \mathrm{meV}$. The same data were taken in the (100) zone, which appear in the righthand portion of Fig. 10. Essentially the same trends are observed in this zone, with the TA mode again becoming almost indistinguishable from background at $300 \mathrm{~K}$. One difference is that the TA mode is better separated at all

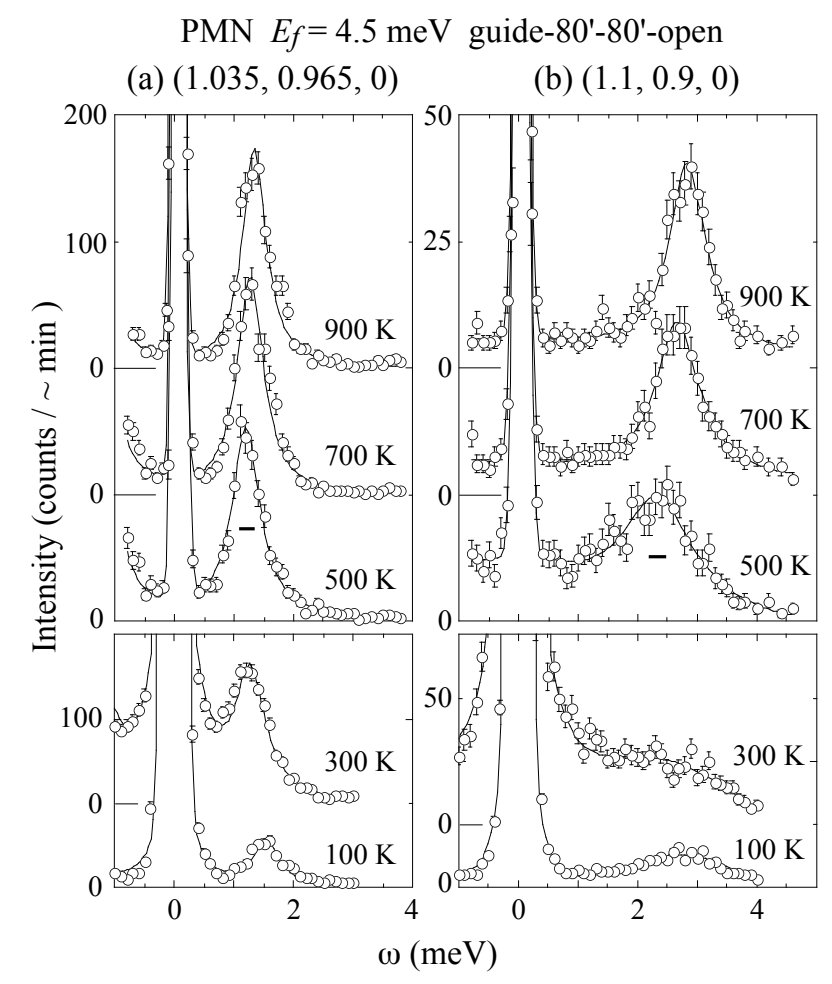

FIG. 9: TA phonon lineshapes in PMN measured on SPINS with cold neutrons at $\vec{Q}=(1.035,0.965,0)$ and $(1.1,0.9,0)$ from $100 \mathrm{~K}$ to $900 \mathrm{~K}$.

temperatures from the steep increase in scattering seen at low energies. These data are consistent with the conjecture of Stock et $a l^{29}$ that the low-energy TA phonon is coupled to the diffuse scattering centered around the elastic position. The inset on the top panel on the righthand side displays the full TA-TO phonon spectrum at $900 \mathrm{~K}$ out to $20 \mathrm{meV}$.

The square of the energies of both the TA and TO modes presented in the two previous figures are plotted versus temperature in Fig. 11. This is done to draw attention to the similarity between the low-energy lattice dynamics of PMN and the behavior expected for a conventional ferroelectric, for which the soft mode frequency squared $\omega^{2}$ varies linearly with $\left(T-T_{c}\right)$. Data for the zone-center soft mode measured at (200) and (220) have been taken from Wakimoto et al. and Stock et al. and added to the top of Fig. 11 for ease of comparison. 27,29 The data taken by Wakimoto et al. are based on energy scans measured at constant- $Q$ at the zone center, whereas Stock et al. determined the zone-center TO energies by extrapolating from data obtained at non-zero $q$. The extrapolation technique permits phonon energies to be extracted in the temperature range where the zonecenter TO mode is heavily damped. These values for the zone-center TO phonon energy have been confirmed by infrared spectroscopy ${ }^{61}$ What this figure immediately reveals, then, is a surprising ferroelectric character of the zone center TO mode above $T_{d}$ and below $T_{c}$, and a corre- 
PMN $E_{f}=14.7 \mathrm{meV} 40^{\prime}-47^{\prime}-40^{\prime}-80^{\prime}$

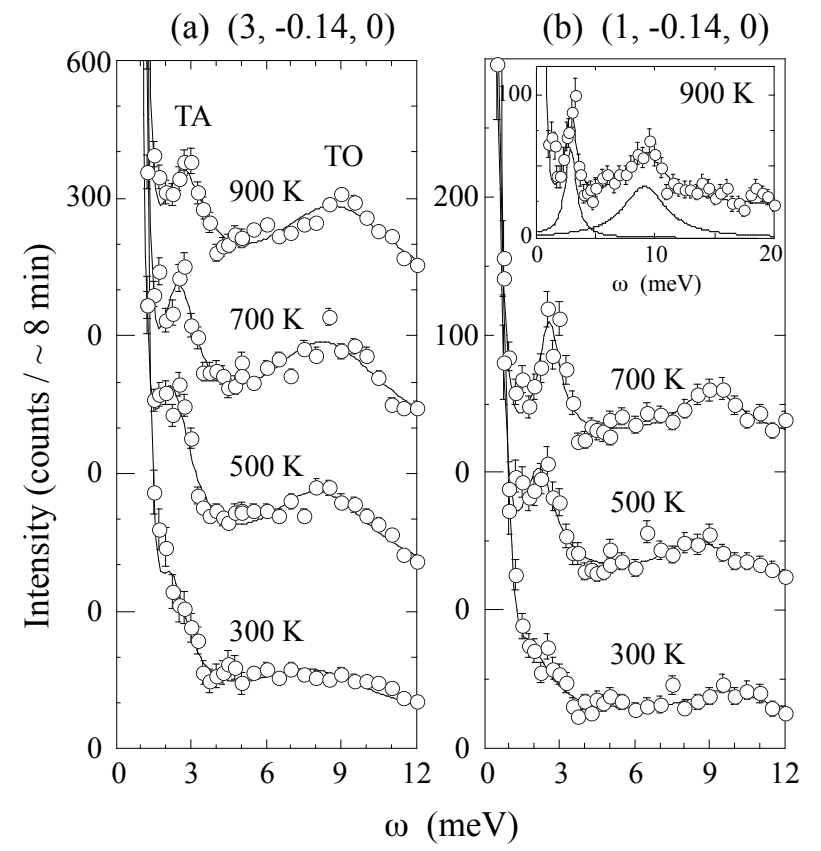

FIG. 10: TA and TO phonon lineshapes in PMN measured on BT9 with thermal neutrons near the waterfall wave vector at $\vec{Q}=(3,-0.14,0)$ and $(1,-0.14,0)$ from $300 \mathrm{~K}$ to $900 \mathrm{~K}$.

sponding change in the behavior of the TA mode energy that is bracketed by the same two temperature scales, which are denoted by the two vertical dash-dotted lines. We note that no such change in the acoustic phonons was observed in PMN-60\%PT where PNR and the associated strong diffuse scattering are absent; therefore the softening of the TA mode is directly related to the development of $\mathrm{PNR} \stackrel{35}{\mathrm{~A}}$ further interesting feature is the minimum that is present in all of these data near $420 \mathrm{~K}$, which is the same temperature at which the strong diffuse scattering first appears when measured with high energy resolution, namely the revised value of the Burns temperature. Therefore, the onset of the diffuse scattering is directly associated with the softening of the TO mode; this is yet further evidence that it is associated with the formation of static, short-range polar correlations.

It is very important to note that the square of the TA phonon energy measured at $\vec{Q}=(1.1,0.9,0)$, which corresponds to $q \sim 0.14 \mathrm{rlu}$, shows a much more pronounced minimum at $420 \mathrm{~K}$ than does that measured at $\vec{Q}=(1.035,0.965,0)$, which corresponds to $q \sim 0.05 \mathrm{rlu}$. This shows that long-wavelength TA phonons exhibit a much weaker response to the formation of static shortrange, polar correlations. This can be understood in terms of a simple physical picture in which those phonons with wavelengths comparable to the size of the PNR are strongly affected (damped) by the PNR whereas longer wavelength phonons simply average over the PNR and are thus not affected by the presence of static, short-

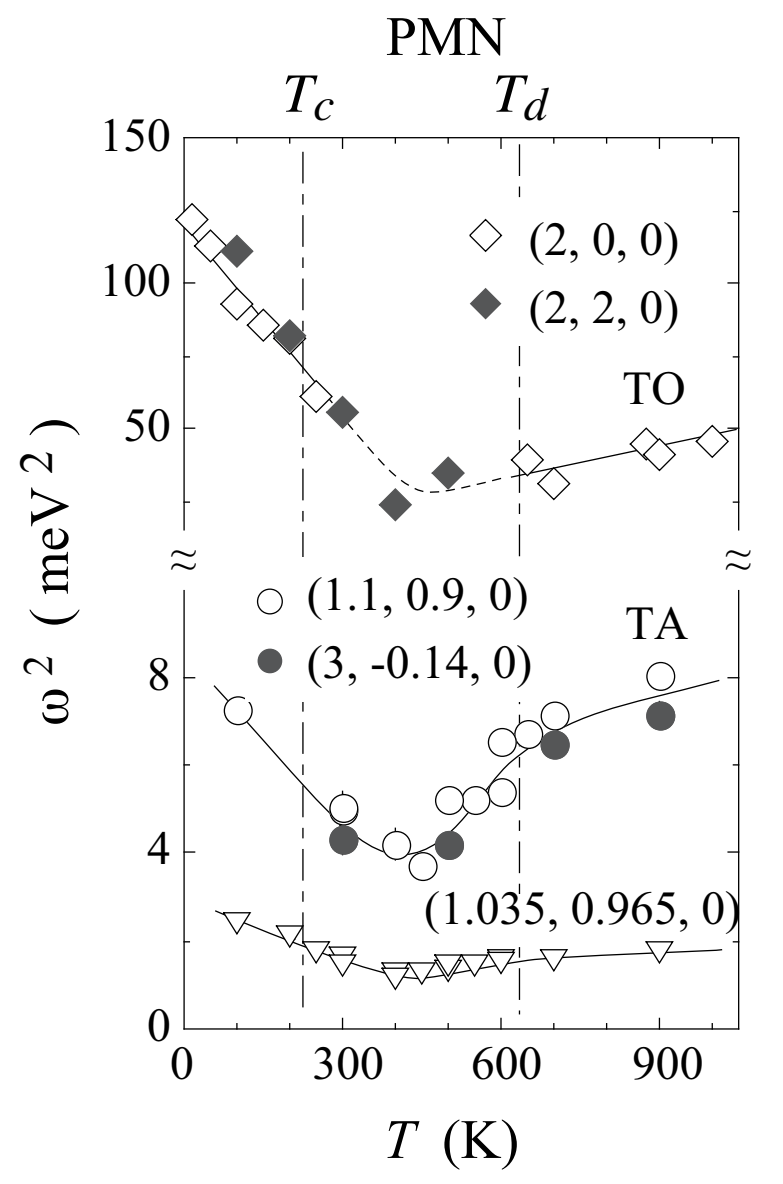

FIG. 11: The square of the phonon energy is plotted versus temperature for the zone-center TO modes measured at (200) (open diamonds) and (220) (solid diamonds), taken from Wakimoto et al. and Stock et al. ${ }^{27,29}$, as well as for three TA modes measured at $(1.035,0.965,0)$ (open triangles), $(1.1,0.9,0)$ (open circles), and $(3,-0.14,0)$ (solid circles). Linear behavior is consistent with that expected for a conventional ferroelectric soft mode.

range polar correlations. This idea was previously proposed to explain the anomalous damping of the TO mode for wave vectors $q \leq q_{w f}$ near the zone center $\stackrel{57}{\text { However, }}$ no strong diffuse scattering is seen in PMN-60\% PT and thus no PNR are present, even though the anomalous TO broadening is still observed; hence this TO broadening, which gives rise to the waterfall effect, cannot be associated with the presence of static, short-range polar correlations. On the other hand, the idea that the acoustic phonons are affected by PNR is confirmed by the absence of any such acoustic phonon broadening in PMN-60\%PT. Thus PNR have a significant effect on the low-energy acoustic phonons over a limited range of reduced wave vectors that may be related to the size of the PNR. In light of the diffuse and inelastic scattering data that have been analyzed so far, we now turn to the detailed measurement of the thermal expansion in PMN. 


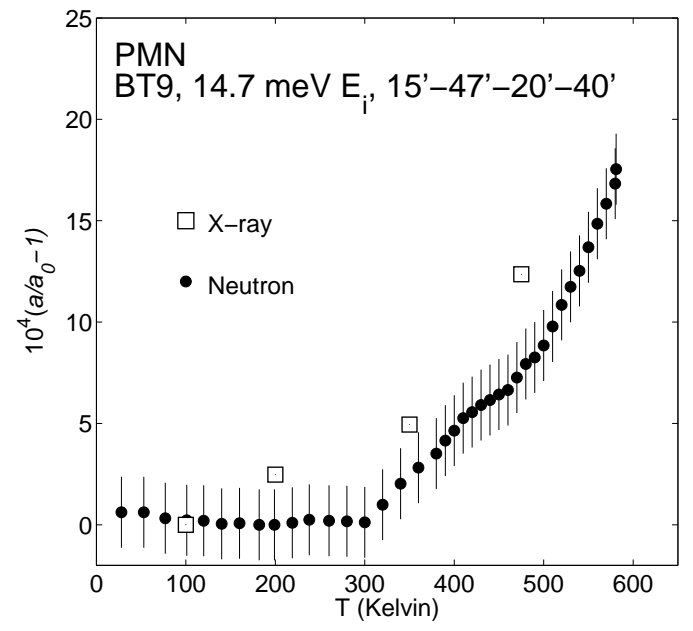

FIG. 12: Lattice strain of single crystal PMN derived from the (220) Bragg reflection measured from $30 \mathrm{~K}$ to $580 \mathrm{~K}$ on BT9. Data were obtained on heating using a perfect $\mathrm{Ge}(004)$ analyzer, an incident neutron energy $E_{i}=14.7 \mathrm{meV}$, and horizontal beam collimations of $15^{\prime}-47^{\prime}-\mathrm{S}-20^{\prime}-40^{\prime}$ to obtain extremely high $q$-resolution. X-ray data from Dkhil et al. are indicated by the open squares for comparison. ${ }^{64}$.

\section{THERMAL EXPANSION: INVAR-LIKE EFFECT BELOW $T_{d}$}

A variety of experimental techniques have been used to measure the thermal expansion in both single crystal and powder samples of PMN, which includes x-ray diffraction, laser dilatometry, and neutron diffraction ${ }^{62,63,64}$ Here we present extremely high $q$-resolution neutron measurements $\left(\delta q \approx 0.0005 \mathrm{rlu}=0.0008 \AA^{-1} \mathrm{HWHM}\right)$ of the cubic unit cell lattice parameter $a$ of PMN crystal \#5 using the special triple-axis configuration described in the experimental section. This configuration, which employs a perfect single crystal of Ge as analyzer, also provides an exceptional energy resolution $\delta E \approx 5-10 \mu \mathrm{eV}$ HWHM. The resulting data are plotted in Fig. 12 in terms of the strain $a / a_{0}-1$, where $a_{0}$ is the lattice parameter at $200 \mathrm{~K}$. Measurements were made on heating from $30 \mathrm{~K}$ to $580 \mathrm{~K}$ using a closed-cycle ${ }^{4} \mathrm{He}$ refrigerator after having first cooled to $30 \mathrm{~K}$. X-ray data measured by Dkhil et al. $\underline{64}$ on a single crystal of PMN, shown as open squares, are provided for comparison.

Several features are interesting to note. First, at low temperature the system exhibits an invar-like effect in which the cubic lattice parameter changes by less than $0.001 \AA$; indeed, the data below $320 \mathrm{~K}$ are consistent with a thermal expansion of zero. At higher temperatures, however, the average thermal expansion is $2.5 \times 10^{-5} 1 / \mathrm{K}$. That these disparate regions of null and high rates of thermal expansion bracket the revised value for the Burns temperature suggests that a direct connection exists between the onset of static, short-range polar correlations and the structural properties of PMN. This behavior seems to be consistent with that of PZN, which also exhibits an increase in the thermal expansion at temperatures above that where the diffuse scattering first appears .65

There is ample evidence of similar behavior reported by other groups in samples of PMN and PMN- $x$ PT. A low-temperature invar-like effect was observed in single crystal PMN-10\%PT, where a transition to a high rate of thermal expansion was found at $400 \mathrm{~K}$; the thermal expansion for this sample at high temperature is $1 \times 10^{-5} 1 / \mathrm{K}$, which is very close to that measured here $\underline{\underline{66}}$ The x-ray study of ceramic samples of PMN by King et al. also show a transition between low and high rates of thermal expansion, but larger values for both $\underline{67} \mathrm{X}$-ray and neutron work conducted by Bonneau et al. on PMN powders yielded onset temperatures and values for the thermal expansion consistent with our single crystal measurements $\stackrel{68}{\underline{6}}$ Finally, an invar effect was also observed in the laser dilatometry study by Arndt and Schmidt on a ceramic sample of PMN, which covered a range from $300 \mathrm{~K}$ to $800 \mathrm{~K} .63$

Even though there is a general trend towards a larger thermal expansion for temperatures above that where the diffuse scattering is onset, there is some sample dependence and some differences between powders and single crystals. As noted by Ye et al.,$\frac{69}{}$ powder measurements of PMN yield a different slope for the thermal expansion measurements than do those of Dkhil et al $\underline{64}$ Also, studies using neutron strain scanning techniques found different thermal expansion coefficients as a function of

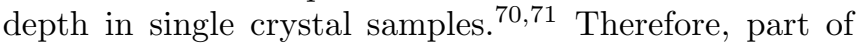
the discrepancy observed between different samples may be associated with surface effects. In this regard, we also note the presence of a change in the slope of the strain curve between $400 \mathrm{~K}$ and $500 \mathrm{~K}$; since this change is not observed in other PMN studies, we believe this feature to be sample-dependent and thus extrinsic.

The phase-shifted model of polar nanoregions proposed by Hirota et al. provides a plausible starting point from which to understand the anomalous invar-like behavior in PMN below the Burns temperature $T_{d}$. This model is based on the observation that the $\mathrm{Pb}, \mathrm{Mg}, \mathrm{Nb}$, and $\mathrm{O}$ ionic displacements obtained from the diffuse scattering measurements of Vakhrushev et al $\stackrel{40}{=}$ on PMN can be decomposed into a center-of-mass conserving component, consistent with the condensation of a soft, transverse optic mode, and a uniform scalar component, corresponding to an acoustic phase shift. In so doing Hirota et al. were able to reconcile the discrepancies between the structure factors of the diffuse scattering and the soft TO modes and, in particular, to explain the weakness of the diffuse scattering intensity observed in the vicinity of the (200) Bragg peak $\frac{52}{2}$ The idea that the PNR are uniformly displaced with respect to the underlying cubic lattice in a direction parallel to the PNR polarization has already been used by Gehring et al. to explain the persistence of the strong diffuse scattering in a single crystal of PZN-8\%PT against large electric fields applied 
along [001] $\stackrel{24}{2}$ In that study it was shown that the diffuse scattering near (003), which measures the projections of the ionic displacements along [001], decreases under an external [001] field as expected. By contrast, the diffuse scattering near (300), which measures the projections of the ionic displacements along [100], i. e. perpendicular to the field direction, remains unaffected even for field strengths up to $10 \mathrm{kV} / \mathrm{cm}$. Thus a uniform polar state is not achieved. This surprising behavior can be understood if one assumes that the electric field applied along [001] removes or reduces the PNR shifts along [001] while preserving those along [100]. The diffuse scattering should then decrease anisotropically with field, as is observed. In the context of the invar-like behavior of PMN below $T_{d}$, we speculate that such uniform shifts of the PNR could effectively stabilize the lattice against subsequent thermal expansion at lower temperature. Such a scenario is consistent with the fact that both $\mathrm{PMN}-10 \% \mathrm{PT}$ and PMN-20\%PT retain cubic structures down to low temperature when examined with high $q$-resolution neutron diffraction methods $\underline{66,72}$

\section{DISCUSSION AND CONCLUSIONS}

We have presented a comprehensive neutron study of the diffuse scattering in the relaxor PMN. We have greatly extended the timescales of previous neutron measurements by taking data on three different spectrometers, BT9, SPINS, and the HFBS, which provide elastic energy resolutions of $500 \mu \mathrm{eV}, 120 \mu \mathrm{eV}$, and $0.4 \mu \mathrm{eV}$ HWHM, respectively. While the backscattering data represent a 300-fold improvement in energy resolution over those obtained by Hiraka et al $\stackrel{28}{\underline{28}}$ on SPINS, both yield an onset temperature of $420 \pm 20 \mathrm{~K}$ for the diffuse scattering. This indicates that the PNR in PMN are static on timescales of at least $2 \mathrm{~ns}$ below $420 \mathrm{~K}$, but are apparently dynamic at higher temperatures. Thus the true Burns temperature in PMN, which was originally interpreted by Burns and Dacol as the condensation temperature of static regions of local, randomly-oriented, nanometerscale polarization,,$\underline{5}$ is $420 \pm 20 \mathrm{~K}$, not $620 \mathrm{~K}$. Independent evidence of the existence of purely dynamic, short-range, polar correlations has been reported in $\mathrm{PMN}-55 \% \mathrm{PT}$, a composition with no strong diffuse scattering and thus no static PNR, by Ko et al. who observed phenomena that are typically related to the relaxation of dynamic PNR $\underline{73}$ These include significant Brillouin quasielastic scattering, a softening of the longitudinal acoustic phonon mode, and a deviation of the dielectric constant from CurieWeiss behavior over an $80 \mathrm{~K}$ temperature interval above $T_{c}$.

Previous measurements of the temperature dependence of the neutron diffuse scattering have been extended in this study to $900 \mathrm{~K}$, well above $T_{d}$. In so doing we have unambiguously established the existence of two types of diffuse scattering based on the observation of two markedly different temperature dependencies, one of which vanishes at $T_{d}$ and one of which does not. We associate the strong, temperature-dependent, diffuse scattering with the formation of static, short-range, polar correlations (PNR) because of its well documented response to an external electric field, and because it first appears at the same temperature at which the soft (polar) TO mode reaches a minimum in energy. We associate the weak, temperature-independent, diffuse scattering, which shows no obvious change across either $T_{c}$ or $T_{d}$, with chemical short-range order because it persists to extremely high temperature. We further confirm the observations of Hiraka et al. $\frac{28}{2}$ who first characterized the distinctly different reciprocal space geometries of both types of diffuse scattering, and we show that the bow-tie shape of the weak diffuse scattering persists up to $900 \mathrm{~K}$ $\gg T_{d}$.

Key effects of the strong, temperature-dependent, diffuse scattering, and thus of the PNR, on the low-energy lattice dynamics of PMN are also highlighted in this study. The neutron inelastic measurements on PMN$60 \%$ PT by Stock et al. prove conclusively that PNR cannot be the origin of the anomalous broadening of longwavelength TO modes observed in PMN, PZN, and other compounds, also known as the waterfall effect, because PMN-60\%PT exhibits the same effect but no strong diffuse scattering (no PNR) ${ }^{35}$ By contrast, many studies have shown that PNR do broaden the TA modes in PMN $14,15,29$ and in PZN- $x \mathrm{PT}, \underline{42}$ but that such effects are absent in compositions with no PNR such as PMN-60\%PT $\stackrel{35}{=}$ Our cold neutron data show that these effects are $q$-dependent. Whereas long-wavelength TA modes with reduced wave vectors $q \ll 0.2 \AA^{-1}$ remain well-defined and exhibit a nearly constant energy width (lifetime) from $100 \mathrm{~K}$ to $900 \mathrm{~K}$, shorter wavelength TA modes with reduced wave vectors $q \approx 0.2 \AA^{-1}$ broaden substantially, with the maximum broadening occurring at $T_{d}=420 \mathrm{~K}$. This result motivates a very simple physical picture in which only those acoustic phonons having wavelengths comparable to the size of the PNR are significantly scattered by the PNR; acoustic modes with wavelengths much larger than the PNR are largely unaffected because they simply average over the PNR. Models describing this effect have been discussed elsewhere ${ }^{29}$ In particular, very recent work by $\mathrm{Xu}$ et al. has revealed the presence of a phase instability in PZN-4.5\% PT that is directly induced by such a PNR-TA phonon interaction. This is shown to produce a pronounced softening and broadening of TA modes in those zones where the diffuse scattering is strong, and provides a natural explanation of the enormous piezoelectric coupling in relaxor materials $\underline{\underline{74}}$

In addition, we have performed neutron measurements of the thermal expansion with extremely high $q$ - and $\hbar \omega$ resolution over a broad temperature range extending well below $T_{c}$ and far above $T_{d} \sim 420 \mathrm{~K}$. In agreement with many other studies, our single crystal samples of PMN exhibit little or no thermal expansion below $T_{d}$, behavior that is reminiscent of the invar effect, but an unusu- 
ally large thermal expansion coefficient of $2.5 \times 10^{-5} 1 / \mathrm{K}$ above $T_{d}$, where the strong diffuse scattering is absent. The crossover between null and large coefficients of thermal expansion coincides closely with $T_{d}$, which suggests that the appearance of static PNR strongly affects the thermal expansion in PMN and thus provides a structural signature of the formation of short-range, polar correlations in zero field. The model of uniformly displaced, or phase-shifted, PNR proposed by Hirota et al., which successfully predicts the anisotropic response of the strong diffuse scattering to an electric field, offers a simplistic, yet plausible, framework in which to understand this anomalous behavior.

Finally, it is satisfying to note that the revised value of $T_{d}=420 \pm 20 \mathrm{~K}$ is consistent with the dielectric susceptibility data of Viehland et al., from which a CurieWeiss temperature $\Theta=398 \mathrm{~K}$ was derived ${ }^{75}$ Such good agreement between $T_{d}$ and $\Theta$ solidifies our identification of the strong diffuse scattering with the condensation of the soft TO mode, which reaches a minimum frequency at $T_{d}=420 \mathrm{~K}$. However this begs the question of how one should interpret the original Burns temperature of $\sim 620 \mathrm{~K}$. At present there are two broadly divergent opinions on this issue, one of which considers $\sim 620 \mathrm{~K}$ to be a meaningful temperature scale in PMN, and one of which does not. As it turns out, this debate is closely tied to another on how many temperature scales are needed to describe the physics of relaxors. We offer no final resolution to this discussion. Instead, we close our paper with a brief summary of the primary studies supporting these contrasting points of view, which is by no means comprehensive, so that the readers may draw their own conclusions.

A number of experimental studies report evidence of either structural or dynamic changes in PMN in the temperature range $600 \mathrm{~K}-650 \mathrm{~K}$, starting with the optical index of refraction measurements of Burns and Dacol. $\stackrel{5}{5}$ Siny and Smirnova were the first to observe strong, first-order Raman scattering in PMN at high temperatures, which, being forbidden in centrosymmetric crystals, implied the presence of some type of lattice distortion ${ }^{76}$ In 2002 Vakhrushev and Okuneva calculated the probability density function for the $\mathrm{Pb}$ ion in PMN within the framework of the spherical layer model using $x$-ray powder diffraction data $\stackrel{77}{ }$ It was shown that this probability density evolves from a single Gaussian function centered on the perovskite $A$-site to a double Gaussian form between $635 \mathrm{~K}$ and $573 \mathrm{~K}$, and that the positions of maximum density for the lead ion follow a power law $\left(T_{d}-T\right)^{0.31}$ with $T_{d}=635 \mathrm{~K}$. This picture was developed further by Prosandeev et al. within a model that ascribed the changes in the lead probability density function and subtle variations in the thermal expansion near $620 \mathrm{~K}$ to a crossover from soft-mode to order-disorder dynamics. ${ }^{78}$ In 2004 Egami et al. first proposed that at very high temperatures the vibrations of the oxygen octahedra are sufficiently faster than those of the $\mathrm{Pb}$ ions that, on average, a high-symmetry, local environment is obtained, whereas at temperatures below $\sim 680 \mathrm{~K}$ the $\mathrm{Pb}$ and $\mathrm{O}$ ions become dynamically correlated ${ }^{79}$ This led to the subsequent reinterpretation of the Burns temperature $(\sim 600 \mathrm{~K})$ as being the point below which dynamic PNR first form, which was based on the dynamic PDF measurements of Dmowski et al. obtained with an elastic energy resolution of $4 \mathrm{meV}$ at six temperatures $(680 \mathrm{~K}, 590 \mathrm{~K}, 450 \mathrm{~K}, 300 \mathrm{~K}, 230 \mathrm{~K}$, and $35 \mathrm{~K}) .80$ Interestingly, after integrating their data from $-5 \mathrm{meV}$ to $+5 \mathrm{meV}$, Dmowski et al. saw evidence of a third temperature scale in PMN of order $300 \mathrm{~K}$, and thus distinct from both $T_{c} \sim 210 \mathrm{~K}$ and $T_{d} \sim 600 \mathrm{~K}$, which they associated with the formation of static PNR. Similar ideas have very recently been put forth by Dkhil et al. based on extremely interesting acoustic emission and thermal expansion measurements, and also in a general summary written by Toulouse..$^{81,82}$

A different approach was taken by Stock et al. who proposed a unified description of the lead-based relaxors on the basis of striking similarities between PMN and the Zn-analogue PZN, both of which display strong, temperature-dependent, diffuse scattering; identical soft mode dynamics; yet no long-range, structural distortion at low temperature in zero field ${ }^{83,84}$ Arguing by analogy with magnetic systems, Stock et al. considered a threedimensional Heisenberg model with cubic anisotropy in the presence of random fields in which the Heisenberg spin corresponds to the local ferroelectric polarization, the cubic anisotropy represents the preferential orientation of the polarization along the $i 111 i$ axes, and the isotropic random magnetic field corresponds to the randomly-oriented local electric fields that originate from the varying charge of the $B$-site cation. Following a suggestion by Aharony, Stock et al. considered the case where the Heisenberg term dominates over the random field term, and the cubic anisotropy term is the weakest of the three. In this picture there would be just two distinct, static, temperature scales. For $T>T_{d}$, the cubic anisotropy is irrelevant and therefore the system should behave like a Heisenberg system in a random field. In this case the excitation spectrum is characterized by Goldstone modes and therefore no long-range order is expected in the presence of random fields 85 Instead the system forms polar nanoregions in a paraelectric background. The second temperature scale $T_{c}$ appears at low temperatures where the cubic anisotropy term becomes important, and in this limit the system should resemble an Ising system in the presence of a random field. This model thus explains the local ferroelectric distortion characterized by the recovery of the soft-optic mode and, although a 3D Ising system in equilibrium should display long-range order in the presence of small random fields, as is observed in magnetic systems, nonequilibrium effects with long time scales become dominant. The presence of such nonequilibrium effects may explain the lack of long-range ferroelectric order in PMN and PZN as well as the history dependence of physical properties such as the linear birefringence. The phase-shifted nature of the 
polar nanoregions may also create another energy barrier against the ordered phase at $T_{c}$. Stock et al. are also able to explain the physics of compounds beyond the MPB, such as PMN-60\%PT, within this model, for which only one temperature scale $\left(T_{c}\right)$ exists. As the Ti concentration Ti increases, the relaxor phase diagram crosses over to a ferroelectric regime, and this can be understood as an increase in the strength of the cubic anisotropy term that is simultaneously accompanied by a reduction of the random fields as must occur in the limit of pure $\mathrm{PbTiO}_{3} \stackrel{35}{ }$ It should be mentioned that other studies have invoked random fields to explain the static and dynamic properties of relaxors $86,87,88$

Independent of the validity of either of the two pictures summarized above, the seminal finding of our study of remains that the strong diffuse scattering in PMN first appears at a temperature that depends sensitively on the energy resolution of the measurement. This fact inevitably raises interesting questions about the significance of the previous value of the Burns temperature $(\sim 620 \mathrm{~K})$. If the strong diffuse scattering in PMN results from the soft TO mode, then other techniques based on $\mathrm{x}$-ray diffraction, thermal neutron scattering, or neutron PDF, which provide comparatively much coarser energy resolution, will be unable to discriminate between lowenergy, dynamic, short-range polar correlations and truly static $(\hbar \omega=0)$ PNR because any low-energy, polar correlations will be integrated into the elastic channel by the broad energy resolution. Thus as the TO mode softens, the associated low-energy, polar, spectral weight will fall within the energy resolution at temperatures above $T_{d}=420 \mathrm{~K}$, and the net result will be an artificially higher value of $T_{d}$ that increases with the size of the energy resolution. This effect should be especially pronounced for phonon modes that are broad in energy (i. e. that have short lifetimes), and this is clearly the case for the soft TO mode in PMN, which exhibits a nearly overdamped lineshape and a minimum frequency precisely at $T_{d}=420 \mathrm{~K}$. While it is true that the structure factor of the strong diffuse scattering is inconsistent with that of the soft TO mode, the phase-shifted model of Hirota et al. provides a way to reconcile this discrepancy. In this respect, we simply suggest that the previous value of $T_{d} \sim 620 \mathrm{~K}$ might not represent a physically meaningful temperature scale in PMN. Future studies examining the values of $T_{d}$ in other relaxor compounds with improved energy resolution are thus of interest.

Our reassessment of the Burns temperature $T_{d}$ immediately clarifies an intimate relationship between the formation of static, short-range polar correlations and the consequent effects on both the low-energy lattice dynamics and structure of PMN. Cold neutron triple-axis and backscattering spectroscopic methods conclusively show the existence of static, short-range polar correlations, only below $T_{d}=420 \pm 20, \mathrm{~K}$ on timescales of at least 2 ns. Thermal neutron measurements of the lattice dynamics reflect the presence of these static PNR through the presence of a distinct minimum in both the soft TO and TA mode energies, both of which occur at $420 \mathrm{~K}$. The effect of PNR on the lattice dynamics is evident only for TA modes having wave vectors of order $0.2 \AA^{-1}$, a fact that could be exploited to determine the size of the PNR. At the same time an enormous change in the coefficient of thermal expansion is seen near $T_{d}$, below which the crystal lattice exhibits invar-like behavior.

\section{ACKNOWLEDGMENTS}

We would like to thank A. Bokov, Y. Fujii, K. Hirota, D. Phelan, S. Shapiro, S. Wakimoto, and G. Xu for stimulating discussions. This study was supported in part by the U. S. - Japan Cooperative NeutronScattering Program, the Natural Sciences and Engineering Research Council (NSERC) of Canada, the National Research Council (NRC) of Canada, the Japanese Ministry of Monbu-Kagaku-shou, RFBR grants 08-02-00908 and $06-02-90088 \mathrm{NSF}$, the U. S. Dept. of Energy under contract No. DE-AC02-98CH10886, the U. S. Office of Naval Research under grant No. N00014-06-1-0166, and by the NSF under grant No. DMR-9986442. We also acknowledge the U. S. Dept. of Commerce, NIST Center for Neutron Research, for providing the neutron scattering facilities used in this study, some of which are supported in part by the National Science Foundation under Agreement No. DMR-0454672.
1 Z.-G. Ye, Key Engineering Materials Vols. 155-156, 81 (1998).

2 S.-E. Park and T. R. Shrout, J. Appl. Phys. 82, 1804 (1997).

3 A.A. Bokov and Z.-G. Ye, J. Mat. Science 41, 31 (2006).

${ }^{4}$ B.E. Vugmeister, Phys. Rev. B 73, 174117 (2006).

5 G. Burns and F. H. Dacol, Solid State Commun. 48, 853 (1983); ibid, Phys. Rev. B 28, 2527 (1983).

6 P. Bonneau, P. Garnier, E. Husson, and A. Morell, Mat. Res. Bull, 24, 201 (1989).

7 N. de Mathan, E. Husson, G. Calvarin, J.R. Gavarri, A.W.
Hewat, and A. Morell J. Phys: Condens Matter 3, 8159 (1991).

8 J. Zhao, A. E. Glazounov, Q. M. Zhang, and B. Toby, Appl. Phys. Lett. 72, 1048 (1998).

${ }^{9}$ K. Hirota, S. Wakimoto, and D.E. Cox, J. Phys. Soc. Jpn. 75, 111006 (2006).

10 R. Blinc, V. Laguta, and B. Zalar, Phys. Rev. Lett. 91, 247601 (2003).

11 V. V. Shvartsman and A. L. Kholkin, Phys. Rev. B 69, 014102 (2004).

12 T. Egami, S. Teslic, W. Dmowski, P. K. Davies, and I.- 
W. Chen, J. Kor. Phys. Soc. 32, S935 (1998); T. Egami, W. Dmowski, S. Teslic, P. K. Davies, I. W. Chen, and H. Chen, Ferroelectrics 206, 231 (1998).

13 I.-K. Jeong, T. W. Darling, J. K. Lee, Th. Proffen, R. H. Heffner, J.S. Park, K.S. Hong, W. Dmowski, and T. Egami, Phys. Rev. Lett. 94, 147602 (2005).

14 A. Naberezhnov, S. B. Vakhrushev, B. Dorner, and H. Moudden, Eur. Phys. J. B 11, 13 (1999).

15 T. Y. Koo, P. M. Gehring, G. Shirane, V. Kiryukhin, S. G. Lee, and S. W. Cheong, Phys. Rev. B 65, 144113 (2002).

16 S. Vakhrushev, A. Naberezhnov, S. K. Sinha, Y. P. Feng, and T. Egami, J. Phys. Chem. Solids 57, 1517 (1996).

17 H. You and Q. M. Zhang, Phys. Rev. Lett. 79, 3950 (1997).

18 G. Xu, G. Shirane, J. R. D. Copley, and P. M. Gehring, Phys. Rev. B 69, 064112 (2004).

19 G. Xu, Z. Zhong, Y. Bing, Z.-G. Ye, and G. Shirane, Nature Materials 4, 887 (2005).

20 M. Matsuura, K. Hirota, P. M. Gehring, Z.-G. Ye, W. Chen, and G. Shirane, Phys. Rev. B 74, 144107 (2006).

${ }^{21}$ G. Xu, Z. Zhong, H. Hiraka, and G. Shirane Phys. Rev. B 70, 174109 (2004).

22 S. B. Vakhrushev, A. A. Naberezhnov, N. M. Okuneva, and B. N. Savenko, Phys. Solid State 40, 1728 (1998).

23 C. Stock, G. Xu, P. M. Gehring, H. Luo, X. Zhao, H. Cao, J. F. Li, D. Viehland, G. Shirane, Phys. Rev. B 76, 064122 (2007).

24 P. M. Gehring, K. Ohwada, and G. Shirane, Phys. Rev. B 70, 014110 (2004).

25 G. Xu, P. M. Gehring, and G. Shirane, Phys. Rev. B 72, 214106 (2005).

26 G. Xu, P. M. Gehring, and G. Shirane, Phys. Rev. B 74, 104110 (2006).

27 S. Wakimoto, C. Stock, R. J. Birgeneau, Z.-G. Ye, W. Chen, W. J. L. Buyers, P. M. Gehring, and G. Shirane, Phys. Rev. B 65, 172105 (2002).

${ }^{28}$ H. Hiraka, S.-H. Lee, P. M. Gehring, G. Xu, and G. Shirane, Phys. Rev. B 70, 184105 (2004).

29 C. Stock, H. Luo, D. Viehland, J. F. Li, I. Swainson, R. J. Birgeneau, and G. Shirane, J. Phys. Soc. Jpn., 74, 3002 (2005).

30 J. Hlinka, S. Kamba, J. Petzelt, J. Kulda, C. A. Randall, and S. J. Zhang, J. Phys. Condens. Matter 15, 4249 (2003).

31 S. N. Gvasaliya, S. G. Lushnikov, and B. Roessli, Cryst. Reports 49, 108 (2004).

32 S. N. Gvasaliya, S. G. Lushnikov, and B. Roessli, Phys. Rev. B 69, 092105 (2004).

33 The study by Hlinka et al. (Ref. 30) employed a much narrower energy resolution of $30 \mu \mathrm{eV}$ full-width at halfmaximum (FWHM) than did the studies of Gvasaliya et al. and Hiraka et al. (200 $\mu \mathrm{eV}$ FWHM) (Ref.'s 32 and 28).

34 B. P. Burton, E. Cockayne, S. Tinte, and U. V. Waghmare, Phase Transitions 79, 91 (2006).

35 C. Stock, D. Ellis, I.P. Swainson, Guangyong Xu, H. Hiraka, Z. Zhong, H. Luo, X. Zhao, D. Viehland, R. J. Birgeneau, and G. Shirane, Phys. Rev. B., 73, 064107 (2006).

36 G. Xu, P. M. Gehring, V. J. Ghosh, and G. Shirane, Acta. Cryst. A60, 598 (2004).

37 A. Meyer, R. M. Dimeo, P. M. Gehring, and D. A. Neumann, Rev. Sci. Instrum., 74, 2759 (2003).

38 W. Chen and Z.-G. Ye, unpublished; Z.-G. Ye, P. Tissot, and H. Schmid, Mater. Res. Bull. 25, 739 (1990).

39 S. B. Vakhrushev, private communication.

40 S. B. Vakhrushev, A. A. Naberezhnov, N. M. Okuneva, and B. N. Savenko, Phys. Solid State 37, 1993 (1995).
41 S. Vakhrushev, A. Ivanov, and J. Kulda, Phys. Chem. Chem. Phys. 7, 2340 (2005).

42 D. La-Orauttapong, J. Toulouse, Z.-G. Ye, W. Chen, R. Erwin, and J. L. Robertson, Phys. Rev. B 67, 134110 (2003).

43 These authors show schematic diffuse scattering intensity contours in Fig. 4 of their paper (no data are shown) for PZN-4.5\%PT and PZN-9\% PT that are wrong because they do not possess the required crystal lattice symmetry.

44 T. R. Welberry, D. J. Goossens, and M. J. Gutmann, Phys. Rev. B 74, 224108 (2006).

45 T.R. Welberry, M.J Gutmann, Hyungje Woo, D. J. Goossens, Guangyong Xu, and C. Stock, J. Appl. Cryst. 38, 639 (2005).

46 S. Wakimoto, G.A. Samara, R.K. Grubbs, E.L. Venturini, L.A. Boatner, G. Xu, G. Shirane, S.-H. Lee, Phys. Rev. B 74, 054101 (2006).

47 M. Pasciak, M. Wolcyrz, and A. Pietraszko, Phys. Rev. B 76, 014117 (2007).

48 B.P Burton and E. Cockayne, Phys. Rev. B 60, R12542 (1999).

49 V.V. Laguta, M.D. Glinchuk, S.N. Nokhrin, I.P. Bykov, R. Blinc, A. Gregorovic, and B. Zalar, Phys. Rev. B 67, 104106 (2003).

50 C. Boulesteix, F. Varnier, A. Llebaria, and E. Husson, J. Solid State. Chem. 108, 141 (1994).

51 O. Svitelskiy, J. Toulouse, G. Yong, and Z.-G. Ye, Phys. Rev. B 68, 104107 (2003).

${ }^{52}$ K. Hirota, Z.-G. Ye, S. Wakimoto, P. M. Gehring, and G. Shirane, Phys. Rev. B 65, 104105 (2002).

53 R. Comes, M. Lambert, and A. Guinier, Acta Crystallogr. Sect. A 26, 244 (1970).

54 S. N. Gvasaliya, B. Roessli, R. A. Cowley, P. Huber, and S. G. Lushnikov, J. Phys. Condens. Matter 17, 4343 (2005).

55 S. Wakimoto, C. Stock, Z.-G. Ye, W. Chen, P. M. Gehring, G. Shirane, Phys. Rev. B 66, 224102 (2002).

56 H. Sompolinsky and A. Zippelius, Phys. Rev B 25, 6860 (1982).

57 P. M. Gehring, S.-E. Park, and G. Shirane, Phys. Rev. Lett. 84, 5216 (2000).

58 P. M. Gehring, S. B. Vakhrushev, and G. Shirane, in Fundamental Physics of Ferroelectrics 2000: Aspen Center for Physics Winter Workshop, edited by R. E. Cohen, AIP Conf. Proc. No. 535 (AIP, New York, 2000), p. 314.

59 P. M. Gehring, S. Wakimoto, Z.-G. Ye, and G. Shirane, Phys. Rev. Lett. 87, 277601 (2001).

60 P. M. Gehring, S.-E. Park, and G. Shirane, Phys. Rev. B 63, 224109 (2001).

61 V. Bovtun, S. Kamba, A. Pashkin, M. Savinov, P. Samoukhina, J. Petzelt, I.P. Bykov, and M.D. Glinchuk, Ferroelectrics, 298, 23 (2004).

62 L. A. Shebanov, P. P. Kapostins, and J. A. Zvirgds, Ferroelectrics 56, 53 (1984).

63 H. Arndt and G. Schmidt, Ferroelectrics 79, 149 (1988).

64 B. Dkhil, J. M. Kiat, G. Calvarin, G. Baldinozzi, S.B. Vakhrushev, and E. Suard, Phys. Rev. B 65, 024104 (2001).

65 D.K. Agrawal, A. Halliyal, and J. Belsick, Mat. Res. Bull. 23, 159 (1988).

66 P. M. Gehring, W. Chen, Z.-G. Ye, and G. Shirane, J. Phys. Condens. Matter 16, 7113 (2004).

67 H. W. King, M. Yildiz, S. H. Ferguson, D. F. Waechter, and S. E. Prasad, Ferroelectric Letters , 55 (2004).

68 P. Bonneau, P. Garnier, G. Calvarin, E. Husson, J. R. 
Gavarri, A.W. Hewat, and A. Morell, J. Sol. State Chem. 91, (1991).

69 Z.-G. Ye, Y. Bing, J. Gao, A. A. Bokov, P. Stephens, B. Noheda, and G. Shirane, Phys. Rev. B 67, 104104 (2003).

${ }^{70}$ K. H. Conlon, H. Luo, D. Viehland, J.F. Li, T. Whan, J.H. Fox, C. Stock, and G. Shirane, Phys. Rev. B 70172204 (2004).

71 G. Xu, P. M. Gehring, C. Stock, K. Conlon, Phase Transitions 79, 135 (2006).

72 G. Xu, D. Viehland, J. F. Li, P. M. Gehring, and G. Shirane, Phys. Rev. B 68, 212410 (2003).

73 J.-H. Ko, S. Kojima, A. A. Bokov, and Z.-G. Ye, Appl. Phys. Lett. 91, 252909 (2007).

74 G. Xu, J. Wen, C. Stock, and P. M. Gehring, Nature Mat. 7, 562 (2008).

75 D. Viehland, S. J. Jang, L. E. Cross, and M. Wuttig, Phys. Rev. B 46, 8003 (1992).

${ }^{76}$ I. G. Siny and T. A. Smirnova, Ferroelectrics 90, 191 (1989).

77 S. B. Vakhrushev and N. M. Okuneva, in Fundamental Physics of Ferroelectrics, edited by R. E. Cohen, AIP Conf. Proc. No. 626 (AIP, New York, 2002), p. 117.

78 http://arxiv.org/ftp/cond-mat/papers/0506/0506132.pdf
79 T. Egami, W. Dmowski, I.-K. Jeong, R. H. Heffner, J.-S. Park, K.-S. Hong, M. Hehlen, and F. Trouw, in Fundamental Physics of Ferroelectrics edited by R. E. Cohen and P. M. Gehring, Williamsburg Conf. Proc. (Washington, DC, 2004), p. 58. (http://people.gl.ciw.edu/cohen/meetings/ferro2004/Ferro2004Abstra

80 W. Dmowski, S. B. Vakhrushev, I.-K. Jeong, M. P. Hehlen, F. Trouw, and T. Egami, Phys. Rev. Lett. 100, 137602 (2008).

81 M. Roth, E. Mojaev, E. Dul'kin, P. Gemeiner, B. Dkhil, Phys. Rev. Lett. 98, 265701 (2007).

82 J. Toulouse, Ferroelectrics 369, 203 (2008).

83 C. Stock, R. J. Birgeneau, S. Wakimoto, J. S. Gardner, W. Chen, Z.-G. Ye, and G. Shirane, Phys. Rev. B 69, 094104 (2004).

${ }^{84}$ G. Xu, Z. Zhong, Y. Bing, Z.-G. Ye, C. Stock, and G. Shirane, Phys. Rev. B 67, 104102 (2003).

85 A. Aharony, private communication.

${ }^{86}$ V. Westphal, W. Kleemann, and M. D. Glinchuk, Phys. Rev. Lett. 68, 847 (1992).

87 R. Pirc and R. Blinc, Phys. Rev. B 60, 13470 (1999).

88 R. Fisch, Phys. Rev. B 67, 094110 (2003). 